\title{
Homotopy theory of modules over operads in symmetric spectra
}

\author{
JOHN E HARPER
}

\begin{abstract}
We establish model category structures on algebras and modules over operads in symmetric spectra and study when a morphism of operads induces a Quillen equivalence between corresponding categories of algebras (resp. modules) over operads.
\end{abstract}

55P43, 55P48; 55U35

\section{Introduction}

Operads parametrize simple and complicated algebraic structures and naturally arise in several areas of algebraic topology, homotopy theory and homological algebra; see Basterra and Mandell [1], Goerss and Hopkins [13], Hinich and Schechtman [18], Kriz and May [24], May [30] and McClure and Smith [31]. The symmetric monoidal category of symmetric spectra (see Hovey, Shipley and Smith [21]) provides a simple and convenient model for the classical stable homotopy category, and is an interesting setting where such algebraic structures naturally arise. Given an operad $\mathcal{O}$ in symmetric spectra, we are interested in the possibility of doing homotopy theory in the categories of $\mathcal{O}$-algebras and $\mathcal{O}$-modules in symmetric spectra, which in practice means putting a Quillen model structure on these categories of algebras and modules. In this setting, $\mathcal{O}_{-}$ algebras are the same as left $\mathcal{O}$-modules concentrated at 0 (Section 3.18). This paper establishes a homotopy theory for algebras and modules over operads in symmetric spectra.

This is the main theorem.

Theorem 1.1 Let $\mathcal{O}$ be an operad in symmetric spectra. Then the category of $\mathcal{O}_{-}$ algebras and the category of left $\mathcal{O}$-modules both have natural model category structures. The weak equivalences and fibrations in these model structures are inherited in an appropriate sense from the stable weak equivalences and the positive flat stable fibrations in symmetric spectra.

Remark 1.2 For ease of notational purposes, we have followed Schwede [37] in using the term flat (eg, flat stable model structure) for what is called $S$ (eg, stable $S$-model structure) in Hovey, Shipley and Smith [21], Schwede [36] and Shipley [39]. 
The theorem remains true when the positive flat stable model structure on symmetric spectra is replaced by the positive stable model structure. This follows immediately from the proof of Theorem 1.1 since every (positive) stable cofibration is a (positive) flat stable cofibration.

Theorem 1.3 Let $\mathcal{O}$ be an operad in symmetric spectra. Then the category of $\mathcal{O}_{-}$ algebras and the category of left $\mathcal{O}$-modules both have natural model category structures. The weak equivalences and fibrations in these model structures are inherited in an appropriate sense from the stable weak equivalences and the positive stable fibrations in symmetric spectra.

In Section 5 we prove that a morphism of operads which is an objectwise stable equivalence induces an equivalence between the corresponding homotopy categories of algebras (resp. modules).

Theorem 1.4 Let $\mathcal{O}$ be an operad in symmetric spectra and let $\mathrm{Alg}_{\mathcal{O}}$ (resp. Lt $\left.\mathrm{Lt}_{\mathcal{O}}\right)$ be the category of $\mathcal{O}$-algebras (resp. left $\mathcal{O}$-modules) with the model structure of Theorem 1.1 or Theorem 1.3. If $f: \mathcal{O} \longrightarrow \mathcal{O}^{\prime}$ is a map of operads, then the adjunctions

$$
\operatorname{Alg}_{\mathcal{O}} \underset{f^{*}}{\stackrel{f_{*}}{\rightleftarrows}} \operatorname{Alg}_{\mathcal{O}^{\prime}}, \quad \operatorname{Lt}_{\mathcal{O}} \underset{f^{*}}{\stackrel{f_{*}}{\rightleftarrows}} \operatorname{Lt}_{\mathcal{O}^{\prime}},
$$

are Quillen adjunctions with left adjoints on top and $f^{*}$ the forgetful functor. If furthermore, $f$ is an objectwise stable equivalence, then the adjunctions (1.5) are Quillen equivalences, and hence induce equivalences on the homotopy categories.

The properties of the flat stable model structure on symmetric spectra are fundamental to the results of this paper. For some of the good properties, see Hovey, Shipley and Smith [21, Theorem 5.3.7 and Corollary 5.3.10]. The positive flat stable model structure, compared to the flat stable model structure, arises very clearly in our arguments. See, for example, Proposition 4.28 and its proof, the following of which is a special case of particular interest.

Proposition 1.6 If $i: X \longrightarrow Y$ is a cofibration between cofibrant objects in symmetric spectra with the positive flat stable model structure and $t \geq 1$, then $X^{\wedge t} \longrightarrow Y^{\wedge t}$ is a cofibration of $\Sigma_{t}$-diagrams in symmetric spectra with the positive flat stable model structure, and hence with the flat stable model structure.

In Section 7 we summarize several constructions and results of particular interest for the special case of algebras over operads. 


\subsection{Relationship to previous work}

One of the theorems of Schwede and Shipley [38] is that the category of monoids in symmetric spectra has a natural model structure inherited from the (flat) stable model structure on symmetric spectra. This result was improved by the author [16] to algebras and left modules over any non $-\Sigma$ operad $\mathcal{O}$ in symmetric spectra.

One of the theorems of Shipley [39] (resp. Mandell, May, Schwede and Shipley [28]) is that the category of commutative monoids in symmetric spectra has a natural model structure inherited from the positive flat stable model structure (resp. positive stable model structure) on symmetric spectra. Theorem 1.1 and Theorem 1.3 improve these results to algebras and left modules over any operad $\mathcal{O}$ in symmetric spectra.

One of the theorems of Elmendorf and Mandell [6] is that for symmetric spectra the category of algebras over any operad $\mathcal{O}$ in simplicial sets has a natural model structure inherited from the positive stable model structure on symmetric spectra. Theorem 1.3 improves this result to algebras and left modules over any operad $\mathcal{O}$ in symmetric spectra. Their proof involves a filtration in the underlying category of certain pushouts of algebras. We have benefitted from their paper and our proofs of Theorem 1.1 and Theorem 1.3 exploit similar filtrations.

Another of the theorems of Elmendorf and Mandell [6] is that a morphism of operads in simplicial sets which is an objectwise weak equivalence induces a Quillen equivalence between categories of algebras over operads. Theorem 1.4 improves this result to algebras and left modules over operads in symmetric spectra.

Our approach to studying algebras and modules over operads is largely influenced by Rezk [35].

Acknowledgments The author would like to thank Bill Dwyer for his constant encouragement and invaluable help and advice. The author is grateful to Emmanuel Farjoun for a stimulating and enjoyable visit to Hebrew University of Jerusalem in spring 2006 and for his invitation which made this possible, and to Paul Goerss and Mike Mandell for helpful comments and suggestions at a Midwest Topology Seminar.

\section{Symmetric spectra}

The purpose of this section is to recall some basic definitions and properties of symmetric spectra. A useful introduction to symmetric spectra is given in the original paper of Hovey, Shipley and Smith [21]; see also the development given by Schwede [37]. Define the sets $\mathbf{n}:=\{1, \ldots, n\}$ for each $n \geq 0$, where $\mathbf{0}:=\varnothing$ denotes the empty set. 
Let $S^{1}$ denote the simplicial circle $\Delta[1] / \partial \Delta[1]$ and for each $n \geq 0$ define $S^{n}:=\left(S^{1}\right)^{\wedge n}$ the $n$-fold smash power of $S^{1}$, where $S^{0}:=\Delta[0]_{+}=\Delta[0] \amalg \Delta[0]$.

Definition 2.1 Let $n \geq 0$.

- $\Sigma_{n}$ is the category with exactly one object $\mathbf{n}$ and morphisms the bijections of sets.

- $\mathrm{S}_{*}$ is the category of pointed simplicial sets and their maps.

- $\mathrm{S}_{*}^{\Sigma_{n}}$ is the category of functors $X: \Sigma_{n} \longrightarrow \mathrm{S}_{*}$ and their natural transformations.

In other words, an object in $\mathrm{S}_{*}^{\Sigma_{n}}$ is a pointed simplicial set $X$ equipped with a basepoint preserving left action of the symmetric group $\Sigma_{n}$ and a morphism in $\mathrm{S}_{*}^{\Sigma_{n}}$ is a map $f: X \longrightarrow Y$ in $\mathrm{S}_{*}$ such that $f$ is $\Sigma_{n}$-equivariant.

\subsection{Symmetric spectra}

Recall the following definition from [21, Section 1.2].

Definition 2.3 A symmetric spectrum $X$ consists of the following:

(1) a sequence of objects $X_{n} \in \mathrm{S}_{*}^{\Sigma_{n}}(n \geq 0)$, and

(2) a sequence of maps $\sigma: S^{1} \wedge X_{n} \longrightarrow X_{n+1}$ in $S_{*}(n \geq 0)$,

such that the iterated maps $\sigma^{p}: S^{p} \wedge X_{n} \longrightarrow X_{n+p}$ are $\Sigma_{p} \times \Sigma_{n}$-equivariant for $p \geq 1$ and $n \geq 0$. Here, $\sigma_{p}:=\sigma\left(S^{1} \wedge \sigma\right) \cdots\left(S^{p-1} \wedge \sigma\right)$ is the composition of the maps

$$
S^{i} \wedge S^{1} \wedge X_{n+p-1-i} \stackrel{S^{i} \wedge \sigma}{\longrightarrow} S^{i} \wedge X_{n+p-i} .
$$

The maps $\sigma$ are the structure maps of the symmetric spectrum.

A map of symmetric spectra $f: X \longrightarrow Y$ is a sequence of maps $f_{n}: X_{n} \longrightarrow Y_{n}$ in $\mathrm{S}_{*}^{\Sigma_{n}}(n \geq 0)$, such that the diagram

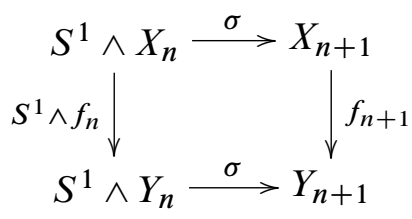

commutes for each $n \geq 0$.

Denote by $\mathrm{Sp}^{\Sigma}$ the category of symmetric spectra and their maps; the null object is denoted by $*$.

The sphere spectrum $S$ is the symmetric spectrum defined by $S_{n}:=S^{n}$, with left $\Sigma_{n}$-action given by permutation and structure maps $\sigma: S^{1} \wedge S^{n} \longrightarrow S^{n+1}$ the natural isomorphisms. 


\subsection{Symmetric spectra as modules over the sphere spectrum}

The purpose of this subsection is to recall the description of symmetric spectra as modules over the sphere spectrum. A similar tensor product construction will appear when working with algebras and left modules over operads in Section 3.

Definition 2.5 Let $n \geq 0$.

- $\Sigma$ is the category of finite sets and their bijections.

- $\mathrm{S}_{*}^{\Sigma}$ is the category of functors $X: \Sigma \longrightarrow \mathrm{S}_{*}$ and their natural transformations.

- If $X \in \mathrm{S}_{*}^{\Sigma}$, define $X_{n}:=X[\mathbf{n}]$ the functor $X$ evaluated on the set $\mathbf{n}$.

- An object $X \in \mathrm{S}_{*}^{\Sigma}$ is concentrated at $n$ if $X_{r}=*$ for all $r \neq n$.

If $X$ is a finite set, define $|X|$ to be the number of elements in $X$.

Definition 2.6 Let $X$ be a finite set and $A$ in $\mathrm{S}_{*}$. The copowers $A \cdot X$ and $X \cdot A$ in $\mathrm{S}_{*}$ are defined as follows:

$$
A \cdot X:=\coprod_{X} A \cong A \wedge X_{+}, \quad X \cdot A:=\coprod_{X} A \cong X_{+} \wedge A,
$$

the coproduct in $\mathrm{S}_{*}$ of $|X|$ copies of $A$.

Definition 2.7 Let $X, Y$ be objects in $\mathrm{S}_{*}^{\Sigma}$. The tensor product $X \otimes Y \in \mathrm{S}_{*}^{\Sigma}$ is the left Kan extension of objectwise smash along coproduct of sets:

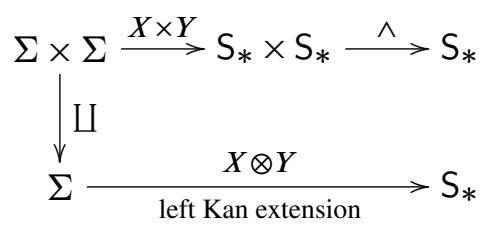

Useful details on Kan extensions and their calculation are given by Mac Lane [26, X], in particular see X.4. The following is a calculation of tensor product, whose proof is left to the reader.

Proposition 2.8 Let $X, Y$ be objects in $\mathrm{S}_{*}^{\Sigma}$ and $N \in \Sigma$, with $n:=|N|$. There are natural isomorphisms

$$
\begin{aligned}
(X \otimes Y)_{n} \cong(X \otimes Y)[N] & \cong \coprod_{\pi: N \underset{\text { in Set }}{\longrightarrow}} X\left[\pi^{-1}(1)\right] \wedge Y\left[\pi^{-1}(2)\right], \\
& \cong \coprod_{n_{1}+n_{2}=n} \Sigma_{n} \underset{\Sigma_{n_{1}} \times \Sigma_{n_{2}}}{.} X_{n_{1}} \wedge Y_{n_{2}} .
\end{aligned}
$$


Remark 2.10 The coproduct is in the category $S_{*}$. Set is the category of sets and their maps.

The following is proved in [21, Section 2.1] and verifies that tensor product in the category $S_{*}^{\Sigma}$ inherits many of the good properties of smash product in the category $S_{*}$.

Proposition 2.11 $\left(\mathrm{S}_{*}^{\Sigma}, \otimes, S^{0}\right)$ has the structure of a closed symmetric monoidal category. All small limits and colimits exist and are calculated objectwise. The unit $S^{0} \in \mathrm{S}_{*}^{\Sigma}$ is given by $S^{0}[\mathbf{n}]=*$ for each $n \geq 1$ and $S^{0}[\mathbf{0}]=S^{0}$.

The sphere spectrum $S$ has two naturally occurring maps $S \otimes S \longrightarrow S$ and $S^{0} \longrightarrow S$ in $\mathrm{S}_{*}^{\Sigma}$ which give $S$ the structure of a commutative monoid in $\left(\mathrm{S}_{*}^{\Sigma}, \otimes, S^{0}\right)$. Furthermore, any symmetric spectrum $X$ has a naturally occurring map $m: S \otimes X \longrightarrow X$ which gives $X$ a left action of $S$ in $\left(\mathrm{S}_{*}^{\Sigma}, \otimes, S^{0}\right)$. The following is proved in [21, Section 2.2] and provides a useful interpretation of symmetric spectra.

Proposition 2.12 Define the category $\Sigma^{\prime}:=\amalg_{n \geq 0} \Sigma_{n}$, a skeleton of $\Sigma$.

(a) The sphere spectrum $S$ is a commutative monoid in $\left(\mathrm{S}_{*}^{\Sigma}, \otimes, S^{0}\right)$.

(b) The category of symmetric spectra is equivalent to the category of left $S$-modules in $\left(\mathrm{S}_{*}^{\Sigma}, \otimes, S^{0}\right)$.

(c) The category of symmetric spectra is isomorphic to the category of left $S$ modules in $\left(\mathrm{S}_{*}^{\Sigma^{\prime}}, \otimes, S^{0}\right)$.

In this paper we will not distinguish between these equivalent descriptions of symmetric spectra.

\subsection{Smash product of symmetric spectra}

The smash product $X \wedge Y \in \mathrm{Sp}^{\Sigma}$ of symmetric spectra $X$ and $Y$ is defined as the colimit

$$
X \wedge Y:=X \otimes S Y:=\operatorname{colim}(X \otimes Y \underset{\text { id } \otimes m}{\stackrel{m \otimes \mathrm{id}}{\longleftarrow}} X \otimes S \otimes Y) .
$$

Note that since $S$ is a commutative monoid, a left action of $S$ on $X$ determines a right action $m: X \otimes S \longrightarrow X$ which gives $X$ the structure of an $(S, S)$-bimodule. Hence the tensor product $X \otimes_{S} Y$ has the structure of a left $S$-module.

The following is proved in [21, Section 2.2] and verifies that smash products of symmetric spectra inherit many of the good properties of smash products of pointed simplicial sets. 
Proposition 2.15 $\left(\mathrm{Sp}^{\Sigma}, \wedge, S\right)$ has the structure of a closed symmetric monoidal category. All small limits and colimits exist and are calculated objectwise.

Recall that by closed we mean there exists a functor which we call mapping object (or function spectrum),

$$
\left(\mathrm{Sp}^{\Sigma}\right)^{\mathrm{op}} \times \mathrm{Sp}^{\Sigma} \longrightarrow \mathrm{Sp}^{\Sigma}, \quad(Y, Z) \longmapsto \operatorname{Map}(Y, Z)
$$

which fits into isomorphisms

$$
\operatorname{hom}(X \wedge Y, Z) \cong \operatorname{hom}(X, \operatorname{Map}(Y, Z))
$$

natural in symmetric spectra $X, Y, Z$. These mapping objects will arise when we introduce mapping sequences associated to circle products in Section 3.

\section{Algebras and modules over operads}

In this section we recall certain definitions and constructions involving symmetric sequences, algebras, and modules over operads. A useful introduction to operads and their algebras is given by Kriz and May [24]. See also the original article of May [30]; other accounts include Berger and Moerdijk [2], Fresse [8], Ginzburg and Kapranov [11], Hinich [17], Markl, Shnider and Stasheff [29], McClure and Smith [32] and Spitzweck [41]. The circle product introduced in Section 3.3 goes back to Getzler and Jones [10] and Smirnov [40] and more recently appears in Fresse [7; 9], Goerss and Hopkins [12], Kapranov and Manin [22], Kelly [23] and Rezk [35]. A fuller account of the material in this section is given in [16] for the general context of a monoidal model category, which was largely influenced by the development in [35].

\subsection{Symmetric sequences}

Definition 3.2 Let $n \geq 0$ and $G$ be a finite group.

- A symmetric sequence in $\mathrm{Sp}^{\Sigma}$ is a functor $A$ : $\Sigma^{\mathrm{op}} \longrightarrow \mathrm{Sp}^{\Sigma}$. SymSeq is the category of symmetric sequences in $\mathrm{Sp}^{\Sigma}$ and their natural transformations; the null object is denoted by $*$.

- SymSeq ${ }^{G}$ is the category of functors $X: G \longrightarrow$ SymSeq and their natural transformations.

- A symmetric sequence $A$ is concentrated at $n$ if $A[\mathbf{r}]=*$ for all $r \neq n$. 


\subsection{Tensor product and circle product of symmetric sequences}

Definition 3.4 Let $X$ be a finite set and $A$ in $\mathrm{Sp}^{\Sigma}$. The copowers $A \cdot X$ and $X \cdot A$ in $\mathrm{Sp}^{\Sigma}$ are defined as follows:

$$
A \cdot X:=\coprod_{X} A \cong A \wedge X_{+}, \quad X \cdot A:=\coprod_{X} A \cong X_{+} \wedge A,
$$

the coproduct in $\mathrm{Sp}^{\Sigma}$ of $|X|$ copies of $A$.

Definition 3.5 Let $A_{1}, \ldots, A_{t}$ be symmetric sequences. The tensor products $A_{1} \check{\otimes} \cdots \check{\otimes} A_{t} \in$ SymSeq are the left Kan extensions of objectwise smash along coproduct of sets:

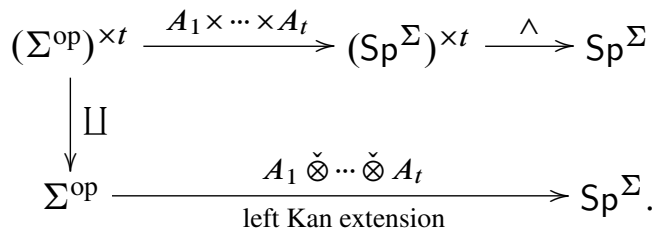

This definition of tensor product in SymSeq is conceptually the same as the definition of tensor product in $S_{*}^{\Sigma}$ given in Definition 2.7. The following is a calculation of tensor product, whose proof is left to the reader.

Proposition 3.6 Let $A_{1}, \ldots, A_{t}$ be symmetric sequences and $R \in \Sigma$, with $r:=|R|$. There are natural isomorphisms

$$
\begin{aligned}
\left(A_{1} \check{\otimes} \cdots \check{\otimes} A_{t}\right)[R] & \cong \coprod_{\pi: R \underset{\text { in Set }}{R} \mathbf{t}} A_{1}\left[\pi^{-1}(1)\right] \wedge \cdots \wedge A_{t}\left[\pi^{-1}(t)\right], \\
& \cong \coprod_{r_{1}+\cdots+r_{t}=r} A_{1}\left[\mathbf{r}_{1}\right] \wedge \cdots \wedge A_{t}\left[\mathbf{r}_{\mathbf{t}}\right]_{\Sigma_{r_{1}} \times \cdots \times \Sigma_{r_{t}}} \Sigma_{r} .
\end{aligned}
$$

It will be useful to extend the definition of tensor powers $A^{\check{\otimes} t}$ to situations in which the integers $t$ are replaced by a finite set $T$.

Definition 3.8 Let $A$ be a symmetric sequence and $R, T \in \Sigma$. The tensor powers $A^{\check{\otimes} T} \in$ SymSeq are defined objectwise by

$$
\begin{aligned}
& \left(A^{\check{\otimes} T}\right)[R]:=\coprod_{\pi: \underset{\text { in } \overrightarrow{\mathrm{Set}}}{\longrightarrow} T} \wedge_{t \in T} A\left[\pi^{-1}(t)\right], \quad T \neq \varnothing,
\end{aligned}
$$

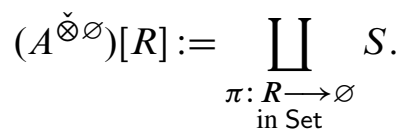


Note that there are no functions $\pi: R \longrightarrow \varnothing$ in Set unless $R=\varnothing$. We will use the abbreviation $A^{\check{\otimes} 0}:=A^{\check{\otimes} \varnothing}$.

Definition 3.10 Let $A, B$ be symmetric sequences, $R \in \Sigma$, and define $r:=|R|$. The circle product (or composition product) $A \circ B \in$ SymSeq is defined objectwise by the coend

$$
(A \circ B)[R]:=A \wedge_{\Sigma}\left(B^{\check{\otimes}-}\right)[R] \cong \coprod_{t \geq 0} A[\mathbf{t}] \wedge_{\Sigma_{t}}\left(B^{\check{\otimes} t}\right)[\mathbf{r}]
$$

Definition 3.12 Let $B, C$ be symmetric sequences, $T \in \Sigma$, and define $t:=|T|$. The mapping sequence $\operatorname{Map}^{\circ}(B, C) \in \operatorname{SymSeq}$ and the mapping object $\operatorname{Map}^{\check{\otimes}}(B, C) \in$ SymSeq are defined objectwise by the ends

$$
\operatorname{Map}^{\circ}(B, C)[T]:=\operatorname{Map}\left(\left(B^{\check{\otimes} T}\right)[-], C\right)^{\Sigma} \cong \prod_{r \geq 0} \operatorname{Map}\left(\left(B^{\check{\otimes} \mathbf{t}}\right)[\mathbf{r}], C[\mathbf{r}]\right)^{\Sigma_{r}},
$$

$$
\operatorname{Map}^{\check{\otimes}}(B, C)[T]:=\operatorname{Map}(B, C[T \amalg-])^{\Sigma} \cong \prod_{r \geq 0} \operatorname{Map}(B[\mathbf{r}], C[\mathbf{t}+\mathbf{r}])^{\Sigma_{r}} .
$$

These mapping sequences and mapping objects are part of closed monoidal category structures on symmetric sequences and fit into isomorphisms

$$
\begin{aligned}
& \operatorname{hom}(A \circ B, C) \cong \operatorname{hom}\left(A, \operatorname{Map}^{\circ}(B, C)\right), \\
& \operatorname{hom}(A \check{\otimes} B, C) \cong \operatorname{hom}\left(A, \operatorname{Map}^{\check{\otimes}}(B, C)\right),
\end{aligned}
$$

natural in symmetric sequences $A, B, C$. The mapping sequences also arise in describing algebras and modules over operads (3.22).

\section{Proposition 3.14}

(a) (SymSeq, $\check{\otimes}, 1)$ has the structure of a closed symmetric monoidal category. All small limits and colimits exist and are calculated objectwise. The unit $1 \in$ SymSeq is given by $1[\mathbf{n}]=*$ for each $n \geq 1$ and $1[\mathbf{0}]=S$.

(b) (SymSeq, o, I) has the structure of a closed monoidal category with all small limits and colimits. Circle product is not symmetric. The (two-sided) unit $I \in$ SymSeq is given by $I[\mathbf{n}]=*$ for each $n \neq 1$ and $I[\mathbf{1}]=S$. 


\subsection{Symmetric sequences build functors}

The category $\mathrm{Sp}^{\Sigma}$ embeds in SymSeq as the full subcategory of symmetric sequences concentrated at 0 , via the functor $\widehat{A}: \mathrm{Sp}^{\Sigma} \longrightarrow$ SymSeq defined objectwise by

$$
\hat{Z}[R]:=\left\{\begin{array}{cl}
Z, & \text { for }|R|=0 \\
*, & \text { otherwise. }
\end{array}\right.
$$

Definition 3.17 Let $\mathcal{O}$ be a symmetric sequence and $Z \in \mathrm{Sp}^{\Sigma}$. The corresponding functor $\mathcal{O}: \mathrm{Sp}^{\Sigma} \longrightarrow \mathrm{Sp}^{\Sigma}$ is defined objectwise by

$$
\mathcal{O}(Z):=\mathcal{O} \circ(Z):=\coprod_{t \geq 0} \mathcal{O}[\mathbf{t}] \wedge \Sigma_{t} Z^{\wedge t} \cong(\mathcal{O} \circ \hat{Z})[\mathbf{0}] .
$$

\subsection{Algebras and modules and over operads}

Definition 3.19 An operad is a monoid object in (SymSeq, o, I) and a morphism of operads is a morphism of monoid objects in (SymSeq, $\circ, I$ ).

Similar to the case of any monoid object, we study operads because we are interested in the objects they act on. A useful introduction to monoid objects and monoidal categories is given in [26, VII].

Definition 3.20 Let $\mathcal{O}$ be an operad. A left $\mathcal{O}$-module is an object in (SymSeq, o, $I$ ) with a left action of $\mathcal{O}$ and a morphism of left $\mathcal{O}$-modules is a map in SymSeq which respects the left $\mathcal{O}$-module structure.

Each operad $\mathcal{O}$ determines a functor $\mathcal{O}: \mathrm{Sp}^{\Sigma} \longrightarrow \mathrm{Sp}^{\Sigma}$ (Definition 3.17) together with natural transformations $m: \mathcal{O O} \longrightarrow \mathcal{O}$ and $\eta$ : id $\longrightarrow \mathcal{O}$ which give the functor $\mathcal{O}: \mathrm{Sp}^{\Sigma} \longrightarrow \mathrm{Sp}^{\Sigma}$ the structure of a monad (or triple) in $\mathrm{Sp}^{\Sigma}$. One perspective offered in [24, I.3] is that operads determine particularly manageable monads. A useful introduction to monads and their algebras is given in [26, VI]. Recall the following definition from [24, I.2 and I.3].

Definition 3.21 Let $\mathcal{O}$ be an operad. An $\mathcal{O}$-algebra is an object in $\mathrm{Sp}^{\Sigma}$ with a left action of the monad $\mathcal{O}: \mathrm{Sp}^{\Sigma} \longrightarrow \mathrm{Sp}^{\Sigma}$ and a morphism of $\mathcal{O}$-algebras is a map in $\mathrm{Sp}^{\Sigma}$ which respects the left action of the monad $\mathcal{O}: \mathrm{Sp}^{\Sigma} \longrightarrow \mathrm{Sp}^{\Sigma}$.

It is easy to verify that an $\mathcal{O}$-algebra is the same as an object $X \in \mathrm{Sp}^{\Sigma}$ with a left $\mathcal{O}$-module structure on $\hat{X}$, and if $X$ and $X^{\prime}$ are $\mathcal{O}$-algebras, then a morphism of $\mathcal{O}$-algebras is the same as a map $f: X \longrightarrow X^{\prime}$ in $\mathrm{Sp}^{\Sigma}$ such that $\hat{f}: \hat{X} \longrightarrow \hat{X}^{\prime}$ is a 
morphism of left $\mathcal{O}$-modules. In other words, an algebra over an operad $\mathcal{O}$ is the same as a left $\mathcal{O}$-module which is concentrated at 0 .

Giving a symmetric sequence $Y$ a left $\mathcal{O}$-module structure is the same as giving a morphism of operads

$$
m: \mathcal{O} \longrightarrow \operatorname{Map}^{\circ}(Y, Y) .
$$

Similarly, giving an object $X \in \mathrm{Sp}^{\Sigma}$ an $\mathcal{O}$-algebra structure is the same as giving a morphism of operads

$$
m: \mathcal{O} \longrightarrow \operatorname{Map}^{\circ}(\hat{X}, \hat{X}) .
$$

This is the original definition given in [30] of an $\mathcal{O}$-algebra structure on $X$, where $\operatorname{Map}^{\circ}(\hat{X}, \widehat{X})$ is called the endomorphism operad of $X$, and motivates the suggestion in $[24 ; 30]$ that $\mathcal{O}[\mathbf{t}]$ should be thought of as parameter objects for $t$-ary operations.

Definition 3.23 Let $\mathcal{O}$ be an operad.

- $\operatorname{Alg}_{\mathcal{O}}$ is the category of $\mathcal{O}$-algebras and their morphisms.

- $\operatorname{Lt}_{\mathcal{O}}$ is the category of left $\mathcal{O}$-modules and their morphisms.

The category $\mathrm{Alg}_{\mathcal{O}}$ embeds in $\operatorname{Lt}_{\mathcal{O}}$ as the full subcategory of left $\mathcal{O}$-modules concentrated at 0 , via the functor $\widehat{A}: \operatorname{Alg}_{\mathcal{O}} \longrightarrow \operatorname{Lt}_{\mathcal{O}}$ defined objectwise by (3.16).

Proposition 3.24 Let $\mathcal{O}$ be an operad in symmetric spectra. There are adjunctions

$$
\mathrm{Sp}^{\Sigma} \underset{U}{\stackrel{\mathcal{O} \circ(-)}{\rightleftarrows}} \mathrm{Alg}_{\mathcal{O}}, \quad \text { SymSeq } \underset{U}{\stackrel{\mathcal{O} \circ-}{\rightleftarrows}} \mathrm{Lt}_{\mathcal{O}}
$$

with left adjoints on top and $U$ the forgetful functor.

Proof The unit $I$ for circle product is the initial operad, hence there is a unique map of operads $f: I \longrightarrow \mathcal{O}$. The desired adjunctions are the following special cases

$$
\mathrm{Sp}^{\Sigma}=\operatorname{Alg}_{I} \underset{f^{*}}{\stackrel{f_{*}}{\rightleftarrows}} \operatorname{Alg}_{\mathcal{O}}, \quad \text { SymSeq }=\mathrm{Lt}_{I} \underset{f^{*}}{\stackrel{f_{*}}{\rightleftarrows}} \mathrm{Lt}_{\mathcal{O}},
$$

of change of operads adjunctions.

Definition 3.26 Let $C$ be a category. A pair of maps of the form

$$
X_{0} \underset{d_{1}}{\longleftarrow} X_{1}
$$

in $\mathrm{C}$ is called a reflexive pair if there exists $s_{0}: X_{0} \longrightarrow X_{1}$ in $\mathrm{C}$ such that $d_{0} s_{0}=$ id and $d_{1} s_{0}=\mathrm{id}$. A reflexive coequalizer is the coequalizer of a reflexive pair. 
The following proposition is proved in [35, Proposition 2.3.5], and allows us to calculate certain colimits in algebras and modules over operads by working in the underlying category. It is also proved in [16] and is closely related to [5, Proposition II.7.2]. Since it plays a fundamental role in several of the main arguments in this paper, we have included a proof below.

Proposition 3.27 Let $\mathcal{O}$ be an operad in symmetric spectra. Reflexive coequalizers and filtered colimits exist in $\mathrm{Alg}_{\mathcal{O}}$ and $\mathrm{Lt}_{\mathcal{O}}$, and are preserved by the forgetful functors.

First we consider the following proposition which is proved in [35, Lemma 2.3.4]. It is also proved in [16] and follows from the proof of [5, Proposition II.7.2] or the arguments in [12, Section 1] as we indicate below.

\section{Proposition 3.28}

(a) If $A_{-1} \longleftarrow A_{0} \longleftarrow A_{1}$ and $B_{-1} \longleftarrow B_{0} \longleftarrow B_{1}$ are reflexive coequalizer diagrams in SymSeq, then their objectwise circle product

$$
A_{-1} \circ B_{-1} \longleftarrow A_{0} \circ B_{0} \longleftarrow A_{1} \circ B_{1}
$$

is a reflexive coequalizer diagram in SymSeq.

(b) If $A, B: \mathrm{D} \longrightarrow$ SymSeq are filtered diagrams, then objectwise circle product of their colimiting cones is a colimiting cone. In particular, there are natural isomorphisms

$$
\underset{d \in \mathrm{D}}{\operatorname{colim}}\left(A_{d} \circ B_{d}\right) \cong\left(\operatorname{colim}_{d \in \mathrm{D}} A_{d}\right) \circ\left(\operatorname{colim}_{d \in \mathrm{D}} B_{d}\right)
$$

in SymSeq.

Proof Consider part (a). The corresponding statement for smash products of symmetric spectra follows from the proof of [5, Proposition II.7.2] or the argument appearing between Definition 1.8 and Lemma 1.9 in [12, Section 1]. Using this together with (3.9) and (3.11), the statement for circle products easily follows by verifying the universal property of a colimit. Consider part (b). It is easy to verify the corresponding statement for smash products of symmetric spectra, and the statement for circle products easily follows as in part (a). 
Proof of Proposition 3.27 Suppose $A_{0} \longleftarrow A_{1}$ is a reflexive pair in $\mathrm{Lt}_{\mathcal{O}}$ and consider the solid commutative diagram

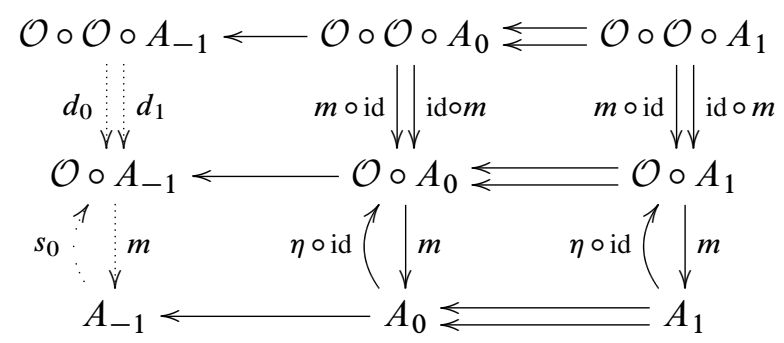

in SymSeq, with bottom row the reflexive coequalizer diagram of the underlying reflexive pair in SymSeq. By Proposition 3.28, the rows are reflexive coequalizer diagrams and hence there exist unique dotted arrows $m, s_{0}, d_{0}, d_{1}$ in SymSeq which make the diagram commute. By uniqueness, $s_{0}=\eta \circ \mathrm{id}, d_{0}=m \circ \mathrm{id}$, and $d_{1}=\mathrm{id} \circ \mathrm{m}$. It is easy to verify that $m$ gives $A_{-1}$ the structure of a left $\mathcal{O}$-module and that the bottom row is a reflexive coequalizer diagram in $\mathrm{Lt}_{\mathcal{O}}$; it is easy to check the diagram lives in $\mathrm{Lt}_{\mathcal{O}}$ and that the colimiting cone is initial with respect to all cones in $\mathrm{Lt}_{\mathcal{O}}$. The case for filtered colimits is similar.

The next proposition is proved in [35, Proposition 2.3.5]. It verifies the existence of all small colimits in algebras and left modules over an operad, and provides one approach to their calculation. The proposition also follows from the argument in [5, Proposition II.7.4]. To keep the paper relatively self-contained, we have included a proof at the end of Section 6.

Proposition 3.29 Let $\mathcal{O}$ be an operad in symmetric spectra. All small colimits exist in $\mathrm{Alg}_{\mathcal{O}}$ and $\mathrm{Lt}_{\mathcal{O}}$. If $A: \mathrm{D} \longrightarrow \mathrm{Lt}_{\mathcal{O}}$ is a small diagram, then colim $A$ in $\mathrm{Lt}_{\mathcal{O}}$ may be calculated by a reflexive coequalizer of the form

$$
\operatorname{colim} A \cong \operatorname{colim}\left(\mathcal{O} \circ\left(\underset{d \in D}{\operatorname{colim}} A_{d}\right) \gtreqless \mathcal{O} \circ\left(\underset{d \in D}{\operatorname{colim}}\left(\mathcal{O} \circ A_{d}\right)\right)\right)
$$

in the underlying category SymSeq; the colimits appearing inside the parenthesis are in the underlying category SymSeq.

The proof of the following is left to the reader.

Proposition 3.30 Let $\mathcal{O}$ be an operad in symmetric spectra. All small limits exist in $\mathrm{Alg}_{\mathcal{O}}$ and $\mathrm{Lt}_{\mathcal{O}}$, and are preserved by the forgetful functors. 


\section{Model structures}

The purpose of this section is to prove Theorem 1.1 and Theorem 1.3, which establish certain model category structures on algebras and left modules over an operad. Model categories provide a setting in which one can do homotopy theory, and in particular, provide a framework for constructing and calculating derived functors. A useful introduction to model categories is given in Dwyer and Spalinski [4]; see also the original articles of Quillen [33; 34] and the more recent by Goerss and Jardine [15], Hirschhorn [19] and Hovey [20]. When we refer to the extra structure of a monoidal model category, we are using Schwede and Shipley [38, Definition 3.1]; an additional condition involving the unit is assumed in Lewis and Mandell [25, Definition 2.3] which we will not require in this paper.

In this paper, our primary method of establishing model structures is to use a small object argument together with the extra structure enjoyed by a cofibrantly generated model category [19, Chapter $11 ; 20$, Section $2.1 ; 38$, Section 2]. The reader unfamiliar with the small object argument may consult [4, Section 7.12] for a useful introduction, followed by the (possibly transfinite) versions described in [19, Chapter 10; 20, Section $2.1 ; 38$, Section 2].

In [38, Section 2] an account of these techniques is provided which will be sufficient for our purposes; our proofs of Theorem 1.1 and Theorem 1.3 will reduce to verifying the conditions of [38, Lemma 2.3(1)]. This verification amounts to a homotopical analysis of certain pushouts (Section 4.3) which lies at the heart of this paper. The reader may contrast this with a path object approach explored in [2], which amounts to verifying the conditions of [38, Lemma 2.3(2)]; compare also [17; 41].

A first step is to recall just enough notation so that we can describe and work with the (positive) flat stable model structure on symmetric spectra, and the corresponding projective model structures on the diagram categories SymSeq and SymSeq ${ }^{G}$, for $G$ a finite group. The functors involved in such a description are easy to understand when defined as the left adjoints of appropriate functors, which is how they naturally arise in this context.

For each $m \geq 0$ and subgroup $H \subset \Sigma_{m}$ denote by $l: H \longrightarrow \Sigma_{m}$ the inclusion of groups and define the evaluation functor $\mathrm{ev}_{m}: \mathrm{S}_{*}^{\Sigma} \longrightarrow \mathrm{S}_{*}^{\Sigma_{m}}$ objectwise by $\operatorname{ev}_{m}(X):=X_{m}$. There are adjunctions

$$
\mathrm{S}_{*} \underset{\lim _{H}}{\longrightarrow} \mathrm{S}_{*}^{H} \underset{l^{*}}{\stackrel{\Sigma_{m} \cdot H}{\gtrless}} \mathrm{S}_{*}^{\Sigma_{m}} \underset{\mathrm{ev} m}{\longrightarrow} \mathrm{S}_{*}^{\Sigma}
$$


with left adjoints on top. Define $G_{m}^{H}: \mathrm{S}_{*} \longrightarrow \mathrm{S}_{*}^{\Sigma}$ to be the composition of the three top functors, and define $\lim _{H} \mathrm{ev}_{m}: \mathrm{S}_{*}^{\Sigma} \longrightarrow \mathrm{S}_{*}$ to be the composition of the three bottom functors; we have dropped the restriction functor $l^{*}$ from the notation. It is easy to check that if $K \in \mathrm{S}_{*}$, then $G_{m}^{H}(K)$ is the object in $\mathrm{S}_{*}^{\Sigma}$ which is concentrated at $m$ with value $\Sigma_{m} \cdot H K$. Consider the forgetful functor $\mathrm{Sp}^{\Sigma} \longrightarrow \mathrm{S}_{*}^{\Sigma}$. It follows from Proposition 2.12 that there is an adjunction

$$
\mathrm{S}_{*}^{\Sigma} \stackrel{S \otimes-}{\gtrless} \mathrm{Sp}^{\Sigma}
$$

with left adjoint on top.

For each $p \geq 0$, define the evaluation functor $\mathrm{Ev}_{p}$ : SymSeq $\longrightarrow \mathrm{Sp}^{\Sigma}$ objectwise by $\operatorname{Ev}_{p}(A):=A[\mathbf{p}]$, and for each finite group $G$, consider the forgetful functor SymSeq $^{G} \longrightarrow$ SymSeq. There are adjunctions

$$
\mathrm{Sp}^{\Sigma} \underset{\operatorname{Ev}_{p}}{\stackrel{G_{p}}{\gtrless}} \text { SymSeq } \stackrel{G \cdot-}{\rightleftarrows} \text { SymSeq }^{G}
$$

with left adjoints on top. It is easy to check that if $X \in \mathrm{Sp}^{\Sigma}$, then $G_{p}(X)$ is the symmetric sequence concentrated at $p$ with value $X \cdot \Sigma_{p}$.

Putting it all together, there are adjunctions

$$
\mathrm{S}_{*} \underset{\lim _{H} \mathrm{ev}_{m}}{\stackrel{G_{m}^{H}}{\rightleftarrows}} \mathrm{S}_{*}^{\Sigma} \stackrel{S \otimes-}{\rightleftarrows} \mathrm{Sp}^{\Sigma} \underset{\mathrm{Ev}_{p}}{\stackrel{G_{p}}{\rightleftarrows}} \text { SymSeq } \stackrel{G \cdot-}{\rightleftarrows} \mathrm{SymSeq}
$$

with left adjoints on top. We are now in a good position to describe several useful model structures. It is proved in [39] that the following two model category structures exist on symmetric spectra.

\section{Definition 4.2}

(a) The flat stable model structure on $\mathrm{Sp}^{\Sigma}$ has weak equivalences the stable equivalences, cofibrations the retracts of (possibly transfinite) compositions of pushouts of maps

$S \otimes G_{m}^{H} \partial \Delta[k]_{+} \longrightarrow S \otimes G_{m}^{H} \Delta[k]_{+} \quad\left(m \geq 0, k \geq 0, H \subset \Sigma_{m}\right.$ subgroup $)$,

and fibrations the maps with the right lifting property with respect to the acyclic cofibrations. 
(b) The positive flat stable model structure on $\mathrm{Sp}^{\Sigma}$ has weak equivalences the stable equivalences, cofibrations the retracts of (possibly transfinite) compositions of pushouts of maps

$S \otimes G_{m}^{H} \partial \Delta[k]_{+} \longrightarrow S \otimes G_{m}^{H} \Delta[k]_{+} \quad\left(m \geq 1, k \geq 0, H \subset \Sigma_{m}\right.$ subgroup $)$,

and fibrations the maps with the right lifting property with respect to the acyclic cofibrations.

It follows immediately from the above description that every positive flat stable cofibration is a flat stable cofibration. Several useful properties of the flat stable model structure are proved in [21, Section 5.3]; here, we remind the reader of Remark 1.2.

The stable model structure on $\mathrm{Sp}^{\Sigma}$ is defined by fixing $H$ in Definition 4.2 (a) to be the trivial subgroup. This is one of several model category structures that is proved in [21] to exist on symmetric spectra.

The positive stable model structure on $\mathrm{Sp}^{\Sigma}$ is defined by fixing $H$ in Definition 4.2(b) to be the trivial subgroup. This model category structure is proved in [28] to exist on symmetric spectra. It follows immediately that every (positive) stable cofibration is a (positive) flat stable cofibration.

These model structures on symmetric spectra enjoy several good properties, including that smash products of symmetric spectra mesh nicely with each of the model structures defined above. More precisely, each model structure above is cofibrantly generated in which the generating cofibrations and acyclic cofibrations have small domains, and that with respect to each model structure $\left(\mathrm{Sp}^{\Sigma}, \wedge, S\right)$ is a monoidal model category. There is also a model structure on $\mathrm{Sp}^{\Sigma}$ which has weak equivalences the stable equivalences and cofibrations the monomorphisms [21, Section 5.3]; this model structure is not a monoidal model structure on $\left(\mathrm{Sp}^{\Sigma}, \wedge, S\right)$.

If $G$ is a finite group, it is easy to check that the diagram categories SymSeq and SymSeq ${ }^{G}$ inherit corresponding projective model category structures, where the weak equivalences (resp. fibrations) are the objectwise weak equivalences (resp. objectwise fibrations). We refer to these model structures by the names above (eg, the positive flat stable model structure on SymSeq ${ }^{G}$ ). Each of these model structures is cofibrantly generated in which the generating cofibrations and acyclic cofibrations have small domains. Furthermore, with respect to each model structure (SymSeq, $\otimes, 1$ ) is a monoidal model category; this is proved in [16], but can easily be verified directly using (3.13).

Proof of Theorem 1.1 Consider SymSeq and $\mathrm{Sp}^{\Sigma}$ both with the positive flat stable model structure. We will prove that the model structure on $\mathrm{Lt}_{\mathcal{O}}\left(\right.$ resp. $\left.\mathrm{Alg} \mathrm{g}_{\mathcal{O}}\right)$ is created 
by the adjunction

$$
\text { SymSeq } \underset{U}{\stackrel{\mathcal{O} \circ-}{\rightleftarrows}} \operatorname{Lt}_{\mathcal{O}} \quad\left(\text { resp. } \quad \mathrm{Sp}^{\Sigma} \underset{U}{\stackrel{\mathcal{O} \circ(-)}{\rightleftarrows}} \operatorname{Alg}_{\mathcal{O}}\right)
$$

with left adjoint on top and $U$ the forgetful functor.

Define a map $f$ in $\operatorname{Lt}_{\mathcal{O}}$ to be a weak equivalence (resp. fibration) if $U(f)$ is a weak equivalence (resp. fibration) in SymSeq. Similarly, define a map $f$ in $A \lg _{\mathcal{O}}$ to be a weak equivalence (resp. fibration) if $U(f)$ is a weak equivalence (resp. fibration) in $\mathrm{Sp}^{\Sigma}$. Define a map $f$ in $\mathrm{Lt}_{\mathcal{O}}\left(\right.$ resp. Alg ${ }_{\mathcal{O}}$ ) to be a cofibration if it has the left lifting property with respect to all acyclic fibrations in $\operatorname{Lt}_{\mathcal{O}}\left(\right.$ resp. $\left.A g_{\mathcal{O}}\right)$.

Consider the case of $\mathrm{Lt}_{\mathcal{O}}$. We want to verify the model category axioms (MC1)-(MC5) in [4]. By Proposition 3.29 and Proposition 3.30, we know that (MC1) is satisfied, and verifying (MC2) and (MC3) is clear. The (possibly transfinite) small object arguments described in the proof of [38, Lemma 2.3] reduce the verification of (MC5) to the verification of Proposition 4.4 below. The first part of (MC4) is satisfied by definition, and the second part of (MC4) follows from the usual lifting and retract argument, as described in the proof of [38, Lemma 2.3]. This verifies the model category axioms. By construction, the model category is cofibrantly generated. Argue similarly for the case of $\mathrm{Alg}_{\mathcal{O}}$ by considering left $\mathcal{O}$-modules concentrated at 0 .

Proof of Theorem 1.3 Consider SymSeq and $\mathrm{Sp}^{\Sigma}$ both with the positive stable model structure. We will prove that the model structure on $\operatorname{Lt}_{\mathcal{O}}\left(\right.$ resp. $\operatorname{Alg}_{\mathcal{O}}$ ) is created by the adjunction

$$
\text { SymSeq } \underset{U}{\stackrel{\mathcal{O} \circ-}{\rightleftarrows}} \operatorname{Lt}_{\mathcal{O}} \quad\left(\text { resp. } \quad \mathrm{Sp}^{\Sigma} \underset{U}{\stackrel{\mathcal{O} \circ(-)}{\rightleftarrows}} \operatorname{Alg}_{\mathcal{O}}\right)
$$

with left adjoint on top and $U$ the forgetful functor. Define a map $f$ in $L_{\mathcal{O}}$ to be a weak equivalence (resp. fibration) if $U(f)$ is a weak equivalence (resp. fibration) in SymSeq. Similarly, define a map $f$ in $\mathrm{Alg}_{\mathcal{O}}$ to be a weak equivalence (resp. fibration) if $U(f)$ is a weak equivalence (resp. fibration) in $\mathrm{Sp}^{\Sigma}$. Define a map $f$ in $\mathrm{Lt}_{\mathcal{O}}$ (resp. $\mathrm{Alg}_{\mathcal{O}}$ ) to be a cofibration if it has the left lifting property with respect to all acyclic fibrations in $\mathrm{Lt}_{\mathcal{O}}$ (resp. $\mathrm{Alg}_{\mathcal{O}}$ ).

The model category axioms are verified exactly as in the proof of Theorem 1.1; (MC5) is verified by Proposition 4.4 below since every cofibration in SymSeq (resp. $\mathrm{Sp}^{\Sigma}$ ) with the positive stable model structure is a cofibration in SymSeq (resp. $\mathrm{Sp}^{\Sigma}$ ) with the positive flat stable model structure. 


\subsection{Homotopical analysis of certain pushouts}

The purpose of this section is to prove the following proposition which we used in the proofs of Theorem 1.1 and Theorem 1.3. The constructions developed here will also be important for homotopical analyses in other sections of this paper.

Proposition 4.4 Let $\mathcal{O}$ be an operad in symmetric spectra, $A \in \mathrm{Lt}_{\mathcal{O}}$, and $i: X \longrightarrow Y$ a generating acyclic cofibration in SymSeq with the positive flat stable model structure. Consider any pushout diagram in $\mathrm{Lt}_{\mathcal{O}}$ of the form

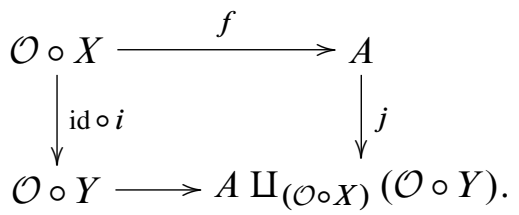

Then $j$ is a monomorphism and a weak equivalence.

Symmetric arrays arise naturally when calculating certain coproducts and pushouts of left modules and algebras over operads (Proposition 4.7 and Proposition 4.20).

\section{Definition 4.6}

- A symmetric array in $\mathrm{Sp}^{\Sigma}$ is a symmetric sequence in SymSeq; ie a functor $A: \Sigma^{\mathrm{op}} \longrightarrow$ SymSeq.

- SymArray $:=$ SymSeq ${ }^{\Sigma^{\mathrm{op}}} \cong\left(\mathrm{Sp}^{\Sigma}\right)^{\Sigma^{\mathrm{op}} \times \Sigma^{\mathrm{op}}}$ is the category of symmetric arrays in $\mathrm{Sp}^{\Sigma}$ and their natural transformations.

First we analyze certain coproducts of modules over operads. The following proposition is proved in [16] in the more general context of monoidal model categories, and was motivated by a similar argument given in [14, Section 2.3] and [27, Section 13] in the context of algebras over an operad. Since the proposition is important to several results in this paper, and in an attempt to keep the paper relatively self-contained, we have included a proof below.

Proposition 4.7 Let $\mathcal{O}$ be an operad in symmetric spectra, $A \in \mathrm{Lt}_{\mathcal{O}}$, and $Y \in \mathrm{SymSeq}_{\text {. }}$ Consider any coproduct in $\mathrm{Lt}_{\mathcal{O}}$ of the form

$$
A \amalg(\mathcal{O} \circ Y) .
$$

There exists a symmetric array $\mathcal{O}_{A}$ and natural isomorphisms

$$
A \amalg(\mathcal{O} \circ Y) \cong \coprod_{q \geq 0} \mathcal{O}_{A}[\mathbf{q}] \check{\otimes}_{\Sigma_{q}} Y^{\check{\otimes} q}
$$


in the underlying category SymSeq. If $q \geq 0$, then $\mathcal{O}_{A}[\mathbf{q}]$ is naturally isomorphic to a colimit of the form

$$
\mathcal{O}_{A}[\mathbf{q}] \cong \operatorname{colim}\left(\coprod_{p \geq 0} \mathcal{O}[\mathbf{p}+\mathbf{q}] \wedge \Sigma_{p} A^{\check{\otimes} p} \underset{d_{0}}{\longleftarrow} \coprod_{p \geq 0} \mathcal{O}[\mathbf{p}+\mathbf{q}] \wedge \Sigma_{p}(\mathcal{O} \circ A)^{\check{\otimes} p}\right),
$$

in SymSeq where $d_{0}$ is induced by operad multiplication and $d_{1}$ is induced by $m: \mathcal{O} \circ A \longrightarrow A$.

Remark 4.9 Other possible notations for $\mathcal{O}_{A}$ include $\mathrm{U}_{\mathcal{O}}(A)$ or $\mathrm{U}(A)$; these are closer to the notation used in $[6 ; 27]$ and are not to be confused with the forgetful functors.

First we make the following observation.

Proposition 4.10 Let $\mathcal{O}$ be an operad in symmetric spectra and $A \in \mathrm{Lt}_{\mathcal{O}}$. Then

$$
A \stackrel{m}{\longleftarrow} \mathcal{O} \circ A \underset{\text { id } \circ m}{\stackrel{m}{\gtrless} \text { id }} \mathcal{O} \circ \mathcal{O} \circ A
$$

is a reflexive coequalizer diagram in $\mathrm{Lt}_{\mathcal{O}}$.

Proof We use a split fork argument. The unit map $\eta: I \longrightarrow \mathcal{O}$ induces a map $s_{0}:=\mathrm{id} \circ \eta \circ \mathrm{id}: \mathcal{O} \circ A \longrightarrow \mathcal{O} \circ \mathcal{O} \circ A$ in $\mathrm{Lt}_{\mathcal{O}}$. Relabeling the three maps in (4.11) as $d_{0}:=m, d_{0}:=m \circ \mathrm{id}, d_{1}:=\mathrm{id} \circ m$, it is easy to verify that $d_{0} s_{0}=\mathrm{id}$ and $d_{1} s_{0}=\mathrm{id}$. Hence the pair of maps is a reflexive pair in $L t_{\mathcal{O}}$, and by Proposition 3.27 it is enough to verify that (4.11) is a coequalizer diagram in the underlying category SymSeq. The unit map $\eta: I \longrightarrow \mathcal{O}$ also induces maps

$$
\begin{aligned}
& s_{-1}:=\eta \circ \mathrm{id}: A \longrightarrow \mathcal{O} \circ A \\
& s_{-1}:=\eta \circ \mathrm{id} \circ \mathrm{id}: \mathcal{O} \circ A \longrightarrow \mathcal{O} \circ \mathcal{O} \circ A
\end{aligned}
$$

in the underlying category SymSeq which satisfy the relations

$$
d_{0} d_{0}=d_{0} d_{1}, \quad d_{0} s_{-1}=\mathrm{id}, \quad d_{1} s_{-1}=s_{-1} d_{0} .
$$

Using these relations, it is easy to check that (4.11) is a coequalizer diagram in SymSeq by verifying the universal property of colimits.

Proof of Proposition 4.7 The objectwise coproduct of two reflexive coequalizer diagrams is a reflexive coequalizer diagram, hence by Proposition 4.10 the coproduct 
(4.8) may be calculated by a reflexive coequalizer in $\mathrm{Lt}_{\mathcal{O}}$ of the form

$$
A \amalg(\mathcal{O} \circ Y) \cong \operatorname{colim}\left((\mathcal{O} \circ A) \amalg(\mathcal{O} \circ Y) \underset{d_{0}}{\longleftarrow}(\mathcal{O} \circ \mathcal{O} \circ A) \amalg(\mathcal{O} \circ Y)\right) .
$$

The maps $d_{0}$ and $d_{1}$ are induced by maps $m: \mathcal{O} \circ \mathcal{O} \longrightarrow \mathcal{O}$ and $m: \mathcal{O} \circ A \longrightarrow A$, respectively. By Proposition 3.27, this reflexive coequalizer may be calculated in the underlying category SymSeq. There are natural isomorphisms

$$
\begin{aligned}
(\mathcal{O} \circ A) \amalg(\mathcal{O} \circ Y) & \cong \mathcal{O} \circ(A \amalg Y) \\
& \cong \coprod_{t \geq 0} \mathcal{O}[\mathbf{t}] \wedge \Sigma_{t}(A \amalg Y)^{\check{\otimes} t} \\
& \cong \coprod_{q \geq 0}\left(\coprod_{p \geq 0} \mathcal{O}[\mathbf{p}+\mathbf{q}] \wedge \Sigma_{p} A^{\check{\otimes} p}\right) \check{\otimes} \Sigma_{q} Y^{\check{\otimes} q},
\end{aligned}
$$

and similarly,

$$
(\mathcal{O} \circ \mathcal{O} \circ A) \amalg(\mathcal{O} \circ Y) \cong \coprod_{q \geq 0}\left(\coprod_{p \geq 0} \mathcal{O}[\mathbf{p}+\mathbf{q}] \wedge \Sigma_{p}(\mathcal{O} \circ A)^{\check{\otimes} p}\right) \check{\otimes}_{\Sigma_{q}} Y^{\check{\otimes} q},
$$

in the underlying category SymSeq. The maps $d_{0}$ and $d_{1}$ similarly factor in the underlying category SymSeq.

Remark 4.12 We have used the natural isomorphisms

$$
(A \amalg Y)^{\check{\otimes} t} \cong \coprod_{p+q=t} \Sigma_{p+q} \cdot \Sigma_{p} \times \Sigma_{q} A^{\check{\otimes} p} \check{\otimes} Y^{\check{\otimes} q}
$$

in the proof of Proposition 4.7.

Definition 4.13 Let $i: X \longrightarrow Y$ be a morphism in SymSeq and $t \geq 1$. Define $Q_{0}^{t}:=$ $X^{\check{\otimes} t}$ and $Q_{t}^{t}:=Y^{\check{\otimes} t}$. For $0<q<t$ define $Q_{q}^{t}$ inductively by the pushout diagrams

$$
\begin{gathered}
\Sigma_{t} \cdot \Sigma_{t-q} \times \Sigma_{q} X^{\check{\otimes}(t-q) \check{\otimes}} Q_{q-1}^{q} \stackrel{\mathrm{pr}_{*}}{\longrightarrow} Q_{q-1}^{t} \\
\downarrow^{i_{*}} \\
\Sigma_{t} \cdot \Sigma_{t-q} \times \Sigma_{q} X^{\check{\otimes}(t-q)} \check{\otimes} Y^{\check{\otimes} q} \\
\downarrow
\end{gathered}
$$

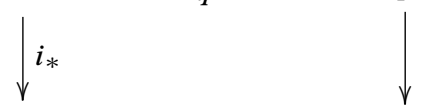

in SymSeq ${ }^{\Sigma_{t}}$. We sometimes denote $Q_{q}^{t}$ by $Q_{q}^{t}(i)$ to emphasize in the notation the map $i: X \longrightarrow Y$. The maps $\mathrm{pr}_{*}$ and $i_{*}$ are the obvious maps induced by $i$ and the appropriate projection maps. 
Remark 4.15 For instance, to construct $Q_{2}^{3}$, first construct $Q_{1}^{2}$ via the pushout diagram

$$
\begin{gathered}
\Sigma_{2} \cdot \Sigma_{1} \times \Sigma_{1} X \check{\otimes} X \longrightarrow \Sigma_{2} \cdot \Sigma_{2} X^{\check{\otimes} 2} \cong X^{\check{\otimes} 2} \\
\qquad \begin{array}{c}
\text { id } \cdot \Sigma_{1} \times \Sigma_{1} \text { id } \check{\otimes} i \\
\Sigma_{2} \cdot \Sigma_{1} \times \Sigma_{1} X \check{\otimes} Y \longrightarrow
\end{array} Q_{1}^{2}
\end{gathered}
$$

in SymSeq ${ }^{\Sigma_{2}}$, then construct $Q_{1}^{3}$ by the pushout diagram

$$
\begin{gathered}
\Sigma_{3} \cdot \Sigma_{2} \times \Sigma_{1} X^{\check{\otimes} 2} \check{\otimes} X \longrightarrow \Sigma_{3} \cdot \Sigma_{3} X^{\check{\otimes} 3} \cong X^{\check{\otimes} 3} \\
\downarrow_{\mathrm{id} \cdot \Sigma_{2} \times \Sigma_{1} \text { id } \check{\otimes} i} \\
\Sigma_{3} \cdot \Sigma_{2} \times \Sigma_{1} X^{\check{\otimes} 2 \check{\otimes} Y}
\end{gathered}
$$

in SymSeq ${ }^{\Sigma_{3}}$, and finally construct $Q_{2}^{3}$ by the pushout diagram

$$
\begin{gathered}
\Sigma_{3} \cdot \Sigma_{1} \times \Sigma_{2} X \check{\otimes} Q_{1}^{2} \stackrel{\mathrm{pr}_{*}}{\longrightarrow} Q_{1}^{3} \\
\downarrow \text { id } \Sigma_{1} \times \Sigma_{2} \text { id } \check{\otimes} i_{*} \\
\Sigma_{3} \cdot \Sigma_{1} \times \Sigma_{2} X \check{\otimes} Y^{\check{\otimes} 2} \longrightarrow
\end{gathered}
$$

in SymSeq ${ }^{\Sigma_{3}}$. The map $i_{*}$ in (4.17) is induced via (4.16) by the two maps

$$
\begin{gathered}
X^{\check{\otimes} 2} \longrightarrow Y^{\check{\otimes} 2}, \\
\Sigma_{2} \cdot \Sigma_{1} \times \Sigma_{1} X \check{\otimes} Y \longrightarrow \Sigma_{2} \cdot \Sigma_{1} \times \Sigma_{1} Y \check{\otimes} Y \longrightarrow \Sigma_{2} \cdot \Sigma_{2} Y^{\check{\otimes} 2} \cong Y^{\check{\otimes} 2} .
\end{gathered}
$$

The pushout diagram

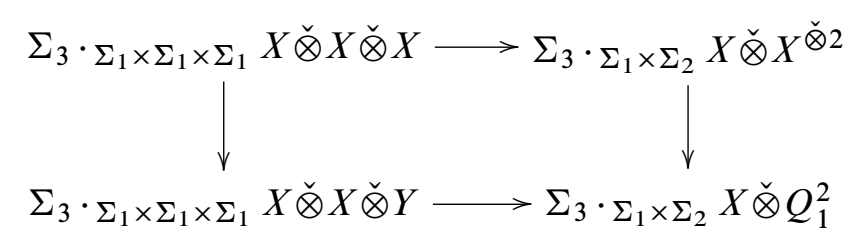

in SymSeq ${ }^{\Sigma_{3}}$ is obtained by applying $\Sigma_{3} \cdot \Sigma_{1} \times \Sigma_{2} X \check{\otimes}-$ to (4.16); the map $\mathrm{pr}_{*}$ in (4.17) is induced via (4.18) by the two maps

$$
\begin{gathered}
\Sigma_{3} \cdot \Sigma_{1} \times \Sigma_{2} X \check{\otimes} X^{\check{\otimes} 2} \longrightarrow \Sigma_{3} \cdot \Sigma_{3} X^{\check{\otimes} 3} \cong X^{\check{\otimes} 3} \longrightarrow Q_{1}^{3}, \\
\Sigma_{3} \cdot \Sigma_{1} \times \Sigma_{1} \times \Sigma_{1} X \check{\otimes} X \check{\otimes} Y \longrightarrow \Sigma_{3} \cdot \Sigma_{2} \times \Sigma_{1} X^{\check{\otimes} 2} \check{\otimes} Y \longrightarrow Q_{1}^{3} .
\end{gathered}
$$


Remark 4.19 The construction $Q_{t-1}^{t}$ can be thought of as a $\Sigma_{t}$-equivariant version of the colimit of a punctured $t$-cube [16]. There is a natural isomorphism $Y^{\check{\otimes} t} / Q_{t-1}^{t} \cong$ $(Y / X)^{\check{\otimes} t}$.

The following proposition is proved in [16] in the more general context of monoidal model categories, and was motivated by a similar construction given in [6, Section 12] in the context of simplicial multifunctors of symmetric spectra. Since several results in this paper require both the proposition and its proof, and in an effort to keep the paper relatively self-contained, we have included a proof below.

Proposition 4.20 Let $\mathcal{O}$ be an operad in symmetric spectra, $A \in \mathrm{Lt}_{\mathcal{O}}$, and $i: X \longrightarrow Y$ in SymSeq. Consider any pushout diagram in $\mathrm{Lt}_{\mathcal{O}}$ of the form

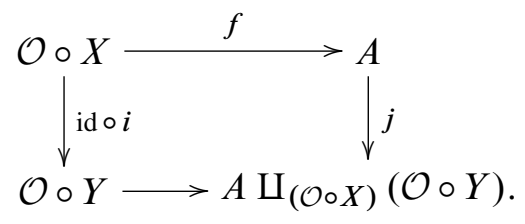

The pushout in (4.21) is naturally isomorphic to a filtered colimit of the form

$$
A \amalg_{(\mathcal{O} \circ X)}(\mathcal{O} \circ Y) \cong \operatorname{colim}\left(A_{0} \stackrel{j_{1}}{\longrightarrow} A_{1} \stackrel{j_{2}}{\longrightarrow} A_{2} \stackrel{j_{3}}{\longrightarrow} \cdots\right)
$$

in the underlying category SymSeq, with $A_{0}:=\mathcal{O}_{A}[\mathbf{0}] \cong A$ and $A_{t}$ defined inductively by pushout diagrams in SymSeq of the form

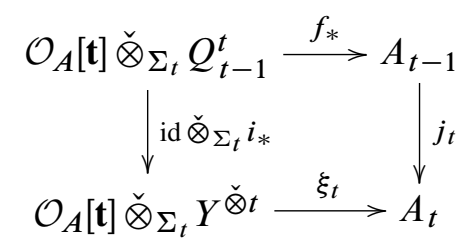

Proof It is easy to verify that the pushout in (4.21) may be calculated by a reflexive coequalizer in $\mathrm{Lt}_{\mathcal{O}}$ of the form

$$
A \amalg_{(\mathcal{O} \circ X)}(\mathcal{O} \circ Y) \cong \operatorname{colim}(A \amalg(\mathcal{O} \circ Y) \underset{\bar{f}}{\longleftarrow} A \amalg(\mathcal{O} \circ X) \amalg(\mathcal{O} \circ Y)) .
$$

By Proposition 3.27, this reflexive coequalizer may be calculated in the underlying category SymSeq. Hence it suffices to reconstruct this coequalizer in SymSeq via a suitable filtered colimit in SymSeq. A first step is to understand what it means to give a cone in SymSeq out of this diagram. 
The maps $\bar{i}$ and $\bar{f}$ are induced by maps ido $i_{*}$ and ido $f_{*}$ which fit into the commutative diagram

$$
\begin{aligned}
& A \amalg(\mathcal{O} \circ(X \amalg Y))<\mathcal{O} \circ(A \amalg X \amalg Y) \underset{d_{1}}{\stackrel{d_{0}}{\leftrightarrows}} \mathcal{O} \circ((\mathcal{O} \circ A) \amalg X \amalg Y)
\end{aligned}
$$

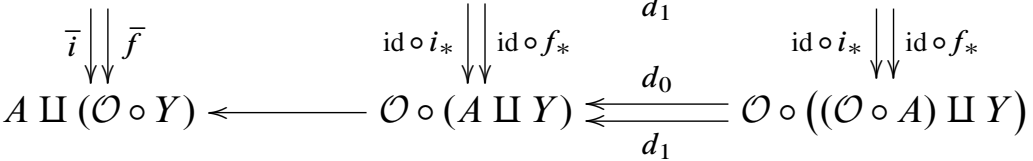

in $\mathrm{Lt}_{\mathcal{O}}$, with rows reflexive coequalizer diagrams, and maps $i_{*}$ and $f_{*}$ in SymSeq induced by $i: X \longrightarrow Y$ and $f: X \longrightarrow A$ in SymSeq. Here we have used the same notation for both $f$ and its adjoint (3.25). By Proposition 3.27, the pushout in (4.21) may be calculated by the colimit of the left-hand column of (4.24) in the underlying category SymSeq. By (4.24) and Proposition 4.7, $f$ induces maps $\bar{f}_{q, p}$ which make the diagrams

$$
\begin{aligned}
& A \amalg(\mathcal{O} \circ(X \amalg Y)) \cong \coprod_{q \geq 0} \coprod_{p \geq 0}() \stackrel{\text { in }_{q, p}}{\longleftarrow}\left(\mathcal{O}_{A}[\mathbf{p}+\mathbf{q}] \check{\otimes}_{\Sigma_{p} \times \Sigma_{q}} X^{\check{\otimes} p} \check{\otimes} Y^{\check{\otimes} q}\right)
\end{aligned}
$$

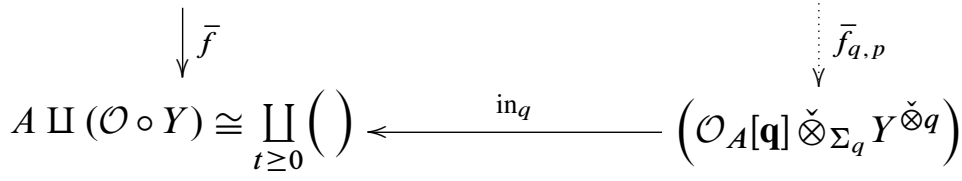

in SymSeq commute. Similarly, $i$ induces maps $\bar{i}_{q, p}$ which make the diagrams

$$
\begin{aligned}
& A \amalg(\mathcal{O} \circ(X \amalg Y)) \cong \coprod_{q \geq 0} \coprod_{p \geq 0}() \stackrel{\text { in }_{q, p}}{\longleftarrow}\left(\mathcal{O}_{A}[\mathbf{p}+\mathbf{q}] \check{\otimes}_{\Sigma_{p} \times \Sigma_{q}} X^{\check{\otimes} p} \check{\otimes} Y^{\check{\otimes} q}\right)
\end{aligned}
$$

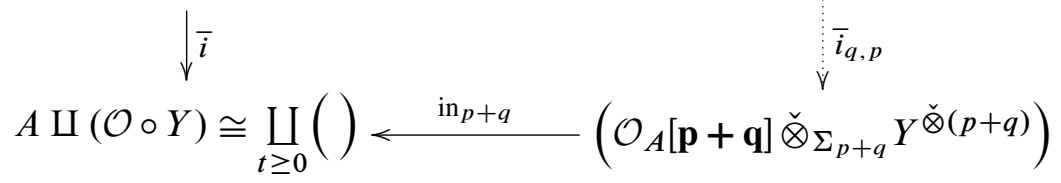

in SymSeq commute. We can now describe more explicitly what it means to give a cone in SymSeq out of the left-hand column of (4.24). Let $\varphi: A \amalg(\mathcal{O} \circ Y) \longrightarrow \cdot$ be a morphism in SymSeq and define $\varphi_{q}:=\varphi \operatorname{in}_{q}$. Then $\varphi \bar{i}=\varphi \bar{f}$ if and only if the diagrams

$$
\mathcal{O}_{A}[\mathbf{p}+\mathbf{q}] \check{\otimes}_{\Sigma_{p} \times \Sigma_{q}} X^{\check{\otimes} p} \check{\otimes} Y^{\check{\otimes} q} \stackrel{\bar{f}_{q, p}}{\longrightarrow} \mathcal{O}_{A}[\mathbf{q}] \check{\otimes}_{\Sigma_{q}} Y^{\check{\otimes} q}
$$

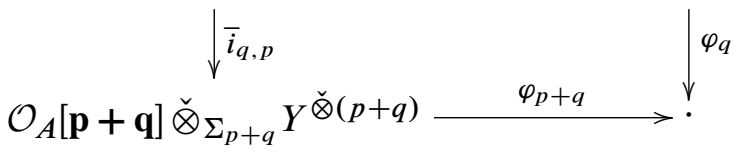


commute for every $p, q \geq 0$. Since $\bar{i}_{q, 0}=$ id and $\bar{f}_{q, 0}=\mathrm{id}$, it is sufficient to consider $q \geq 0$ and $p>0$.

The next step is to reconstruct the colimit of the left-hand column of (4.24) in SymSeq via a suitable filtered colimit in SymSeq. The diagrams (4.25) suggest how to proceed. We will describe two filtration constructions that calculate the pushout (4.21) in the underlying category SymSeq. The purpose of presenting the filtration construction (4.27) is to provide motivation and intuition for the filtration construction (4.23) that we are interested in. Since (4.27) does not use the gluing construction in Definition 4.13 it is simpler to verify that (4.22) is satisfied and provides a useful warm-up for working with (4.23).

For each $t \geq 1$, there are natural isomorphisms

$$
(X \amalg Y)^{\check{\otimes} t}-Y^{\check{\otimes} t} \cong \coprod_{\substack{p+q=t \\ q \geq 0, p>0}} \Sigma_{p+q} \cdot \Sigma_{p} \times \Sigma_{q} X^{\check{\otimes} p} \check{\otimes} Y^{\check{\otimes} q} .
$$

Here, $(X \amalg Y)^{\check{\otimes} t}-Y^{\check{\otimes} t}$ denotes the coproduct of all factors in $(X \amalg Y)^{\check{\otimes} t}$ except $Y^{\check{\otimes} t}$. Define $A_{0}:=\mathcal{O}_{A}[\mathbf{0}] \cong A$ and for each $t \geq 1$ define $A_{t}$ by the pushout diagram

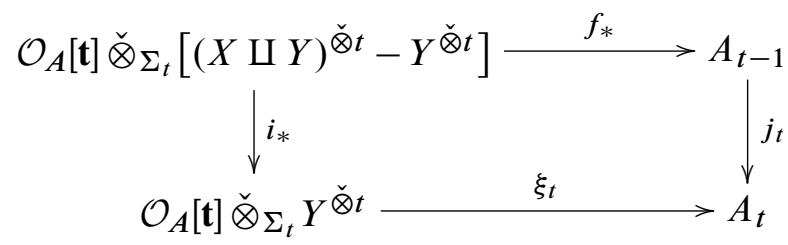

in SymSeq. The maps $f_{*}$ and $i_{*}$ are induced by the appropriate maps $\bar{f}_{q, p}$ and $\bar{i}_{q, p}$. We want to use (4.26), (4.27) and (4.25) to verify that (4.22) is satisfied; it is sufficient to verify the universal property of colimits. By Proposition 4.7, the coproduct $A \amalg(\mathcal{O} \circ Y)$ is naturally isomorphic to a filtered colimit of the form

$$
A \amalg(\mathcal{O} \circ Y) \cong \operatorname{colim}\left(B_{0} \longrightarrow B_{1} \longrightarrow B_{2} \longrightarrow \cdots\right)
$$

in the underlying category SymSeq, with $B_{0}:=\mathcal{O}_{A}[\mathbf{0}]$ and $B_{t}$ defined inductively by pushout diagrams in SymSeq of the form

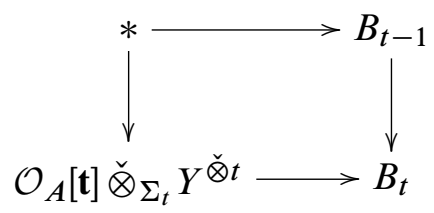


For each $t \geq 1$, there are naturally occurring maps $B_{t} \longrightarrow A_{t}$, induced by the appropriate $\xi_{i}$ and $j_{i}$ maps in (4.27), which fit into the commutative diagram

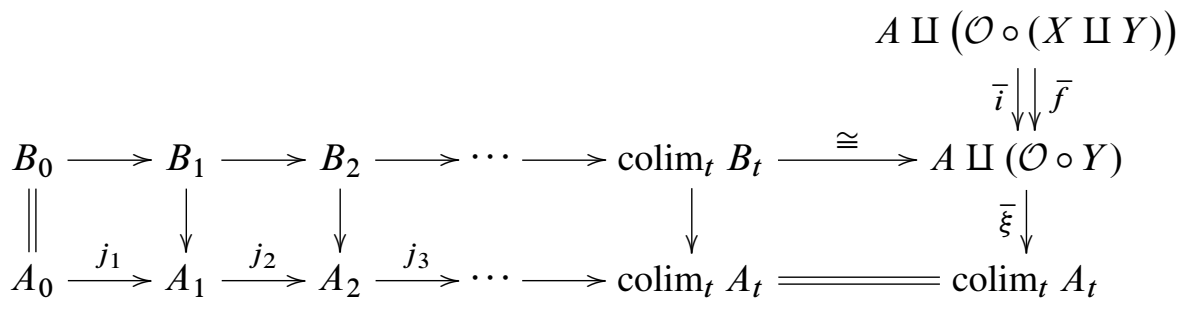

in SymSeq; the morphism of filtered diagrams induces a map $\bar{\xi}$. We claim that the right-hand column is a coequalizer diagram in SymSeq. To verify that $\bar{\xi}$ satisfies $\bar{\xi} \bar{i}=\bar{\xi} \bar{f}$, by (4.25) it is enough to check that the diagram

$$
\begin{aligned}
& \mathcal{O}_{A}[\mathbf{p}+\mathbf{q}] \check{\otimes}_{\Sigma_{p} \times \Sigma_{q}} X^{\check{\otimes} p} \check{\otimes} Y^{\check{\otimes} q} \stackrel{\bar{f}_{q, p}}{\longrightarrow} \mathcal{O}_{A}[\mathbf{q}] \check{\otimes}_{\Sigma_{q}} Y^{\check{\otimes} q} \\
& \downarrow_{\bar{i}_{q, p}} \downarrow \bar{\xi}_{\mathrm{in}_{q}} \\
& \mathcal{O}_{A}[\mathbf{p}+\mathbf{q}] \check{\otimes}_{\Sigma_{p+q}} Y^{\check{\otimes}(p+q)} \stackrel{\bar{\xi} \operatorname{in}_{p+q}}{\longrightarrow} \operatorname{colim}_{t} A_{t}
\end{aligned}
$$

commute for every $q \geq 0$ and $p>0$; this is easily verified using (4.26) and (4.27), and is left to the reader. Let $\varphi: A \amalg(\mathcal{O} \circ Y) \longrightarrow \cdot$ be a morphism in SymSeq such that $\varphi \bar{i}=\varphi \bar{f}$. We want to verify that there exists a unique map $\bar{\varphi}: \operatorname{colim}_{t} A_{t} \longrightarrow \cdot$ in SymSeq such that $\varphi=\bar{\varphi} \bar{\xi}$. Consider the corresponding maps $\varphi_{i}$ in (4.25) and define $\bar{\varphi}_{0}:=\varphi_{0}$. For each $t \geq 1$, the maps $\varphi_{i}$ induce maps $\bar{\varphi}_{t}: A_{t} \longrightarrow \cdot$ such that $\bar{\varphi}_{t} j_{t}=$ $\bar{\varphi}_{t-1}$ and $\bar{\varphi}_{t} \xi_{t}=\varphi_{t}$. In particular, the maps $\bar{\varphi}_{t}$ induce a map $\bar{\varphi}: \operatorname{colim}_{t} A_{t} \longrightarrow \cdot$ in SymSeq. Using (4.25) it is an easy exercise (which the reader should verify) that $\bar{\varphi}$ satisfies $\varphi=\bar{\varphi} \bar{\xi}$ and that $\bar{\varphi}$ is the unique such map. Hence the filtration construction (4.27) satisfies (4.22). One drawback of (4.27) is that it may be difficult to analyze homotopically. A hint at how to improve the construction is given by the observation that the collection of maps $\bar{f}_{q, p}$ and $\bar{i}_{q, p}$ satisfy many compatibility relations. To obtain a filtration construction we can homotopically analyze, the idea is to replace $(X \amalg Y)^{\check{\otimes} t}-Y^{\check{\otimes} t}$ in (4.27) with the gluing construction $Q_{t-1}^{t}$ in Definition 4.13 as follows.

Define $A_{0}:=\mathcal{O}_{A}[\mathbf{0}] \cong A$ and for each $t \geq 1$ define $A_{t}$ by the pushout diagram (4.23) in SymSeq. The maps $f_{*}$ and $i_{*}$ are induced by the appropriate maps $\bar{f}_{q, p}$ and $\bar{i}_{q, p}$. Arguing exactly as above for the case of (4.27), it is easy to use the diagrams (4.25) to verify that (4.22) is satisfied. The only difference is that the naturally occurring maps $B_{t} \longrightarrow A_{t}$ are induced by the appropriate $\xi_{i}$ and $j_{i}$ maps in (4.23) instead of in (4.27). 
The following proposition illustrates some of the good properties of the positive flat stable model structure on SymSeq. The statement in part (b) is motivated by [6, Lemma 12.7] in the context of symmetric spectra with the positive stable model structure. We defer the proof to Section 6 .

Proposition 4.28 Let $B \in \operatorname{SymSeq}^{\Sigma_{t}^{\mathrm{op}}}$ and $t \geq 1$. If $i: X \longrightarrow Y$ is a cofibration between cofibrant objects in SymSeq with the positive flat stable model structure, then

(a) $X^{\check{\otimes} t} \longrightarrow Y^{\check{\otimes} t}$ is a cofibration in SymSeq ${ }^{\Sigma_{t}}$ with the positive flat stable model structure, and hence with the flat stable model structure,

(b) the map $B \check{\otimes}_{\Sigma_{t}} Q_{t-1}^{t} \longrightarrow B \check{\otimes}_{\Sigma_{t}} Y^{\check{\otimes} t}$ is a monomorphism.

We will prove the following proposition in Section 6.

Proposition 4.29 Let $G$ be a finite group and consider SymSeq, SymSeq ${ }^{G}$ and SymSeq ${ }^{\text {op }}$ each with the flat stable model structure.

(a) If $B \in \mathrm{SymSeq}^{G^{\mathrm{op}}}$, then the functor

$$
B \check{\otimes}_{G}-: \text { SymSeq }^{G} \longrightarrow \text { SymSeq }
$$

preserves weak equivalences between cofibrant objects, and hence its total left derived functor exists.

(b) If $Z \in \operatorname{SymSeq}^{G}$ is cofibrant, then the functor

$$
-\check{\otimes}_{G} Z: \text { SymSeq }^{G^{\text {op }}} \longrightarrow \text { SymSeq }
$$

preserves weak equivalences.

We are now in a good position to give a homotopical analysis of the pushout in Proposition 4.4.

Proposition 4.30 If the map $i: X \longrightarrow Y$ in Proposition 4.20 is a generating acyclic cofibration in SymSeq with the positive flat stable model structure, then each map $j_{t}$ is a monomorphism and a weak equivalence. In particular, the map $j$ is a monomorphism and a weak equivalence.

Proof The generating acyclic cofibrations in SymSeq have cofibrant domains. Proposition 4.28 implies that each $j_{t}$ is a monomorphism. We know that $A_{t} / A_{t-1} \cong$ $\mathcal{O}_{A}[\mathbf{t}] \check{\otimes}_{\Sigma_{t}}(Y / X)^{\check{\otimes} t}$ and that $* \longrightarrow Y / X$ is an acyclic cofibration in SymSeq with the positive flat stable model structure. It follows from Proposition 4.28 and Proposition 4.29 that $j_{t}$ is a weak equivalence.

Proof of Proposition 4.4 By assumption, the map $i: X \longrightarrow Y$ is a generating acyclic cofibration in SymSeq with the positive flat stable model structure, hence Proposition 4.30 finishes the proof. 


\section{Relations between homotopy categories}

The purpose of this section is to prove Theorem 1.4, which establishes an equivalence between certain homotopy categories of algebras (resp. modules) over operads. Our argument is a verification of the conditions in [4, Theorem 9.7] for an adjunction to induce an equivalence between the corresponding homotopy categories, and amounts to a homotopical analysis (Section 5.1) of the unit of the adjunction.

Proof of Theorem 1.4 Let $f: \mathcal{O} \longrightarrow \mathcal{O}^{\prime}$ be a morphism of operads and consider the case of left modules. We know (1.5) is a Quillen adjunction since the forgetful functor $f^{*}$ preserves fibrations and acyclic fibrations. Assume furthermore that $f$ is a weak equivalence in the underlying category SymSeq with the positive flat stable model structure; let's verify the Quillen adjunction (1.5) is a Quillen equivalence. By [4, Theorem 9.7], it is enough to verify: for cofibrant $Z \in \mathrm{Lt}_{\mathcal{O}}$ and fibrant $B \in \mathrm{Lt}_{\mathcal{O}^{\prime}}$, a map $\xi: Z \longrightarrow f^{*} B$ is a weak equivalence in $\operatorname{Lt}_{\mathcal{O}}$ if and only if its adjoint map $\eta: f_{*} Z \longrightarrow B$ is a weak equivalence in $\operatorname{Lt}_{\mathcal{O}^{\prime}}$. Noting that $\xi$ factors as

$$
Z \longrightarrow f^{*} f_{*} Z \stackrel{f^{*} \eta}{\longrightarrow} f^{*} B
$$

together with Proposition 5.2 below finishes the proof. Argue similarly for the case of algebras by considering left modules concentrated at 0 .

\subsection{Homotopical analysis of the unit of the adjunction}

The purpose of this subsection is to prove the following proposition which we used in the proof of Theorem 1.4. Our argument is motivated by the proof of [6, Theorem 12.5].

Proposition 5.2 Let $f: \mathcal{O} \longrightarrow \mathcal{O}^{\prime}$ be a morphism of operads and consider $\mathrm{Lt}_{\mathcal{O}}$ with the positive flat stable model structure. If $Z \in \mathrm{Lt}_{\mathcal{O}}$ is cofibrant and $f$ is a weak equivalence in the underlying category SymSeq with the positive flat stable model structure, then the natural map $Z \longrightarrow f^{*} f_{*} Z$ is a weak equivalence in $\operatorname{Lt}_{\mathcal{O}}$.

First we make the following observation.

Proposition 5.3 Consider SymSeq with the positive flat stable model structure. If $W \in$ SymSeq is cofibrant, then the functor

$$
-\circ W: \text { SymSeq } \longrightarrow \text { SymSeq }
$$

preserves weak equivalences. 
Proof Let $A \longrightarrow B$ be a weak equivalence in SymSeq; we want to verify

$$
A[\mathbf{t}] \wedge \Sigma_{t}\left(W^{\check{\otimes} t}\right)[\mathbf{r}] \longrightarrow B[\mathbf{t}] \wedge \Sigma_{t}\left(W^{\check{\otimes} t}\right)[\mathbf{r}]
$$

is a weak equivalence in $\mathrm{Sp}^{\Sigma}$ with the flat stable model structure for each $r, t \geq 0$. By Proposition 4.28 we know $W^{\check{\otimes} t}$ is cofibrant in $\operatorname{SymSeq}^{\Sigma_{t}}$ with the flat stable model structure for each $t \geq 1$. By considering symmetric sequences concentrated at 0 , Proposition 4.29 finishes the proof.

Proof of Proposition 5.2 Let $X \longrightarrow Y$ be a generating cofibration in SymSeq with the positive flat stable model structure, and consider the pushout diagram

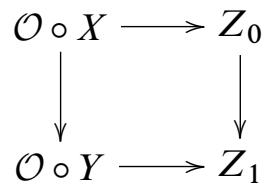

in $\mathrm{Lt}_{\mathcal{O}}$. For each $W \in$ SymSeq consider the natural maps

$$
\begin{aligned}
& Z_{0} \amalg(\mathcal{O} \circ W) \longrightarrow f^{*} f_{*}\left(Z_{0} \amalg(\mathcal{O} \circ W)\right), \\
& Z_{1} \amalg(\mathcal{O} \circ W) \longrightarrow f^{*} f_{*}\left(Z_{1} \amalg(\mathcal{O} \circ W)\right),
\end{aligned}
$$

and note that the left-hand (resp. right-hand) diagram

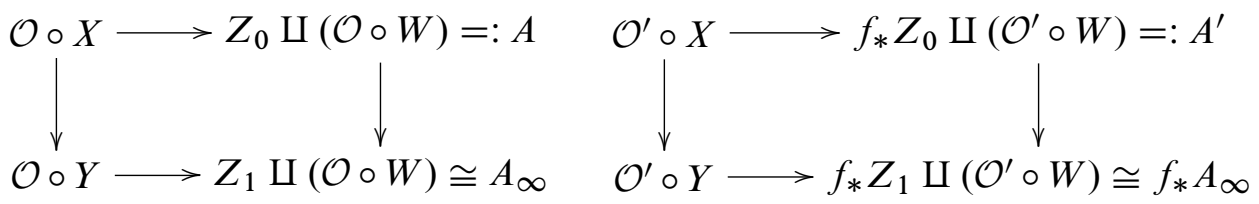

is pushout diagram in $\mathrm{Lt}_{\mathcal{O}}$ (resp. $\mathrm{Lt}_{\mathcal{O}^{\prime}}$ ). Assume (5.5) is a weak equivalence for every cofibrant $W \in$ SymSeq; let's verify (5.6) is a weak equivalence for every cofibrant $W \in$ SymSeq. Suppose $W \in$ SymSeq is cofibrant. By Proposition 4.20 there are corresponding filtrations

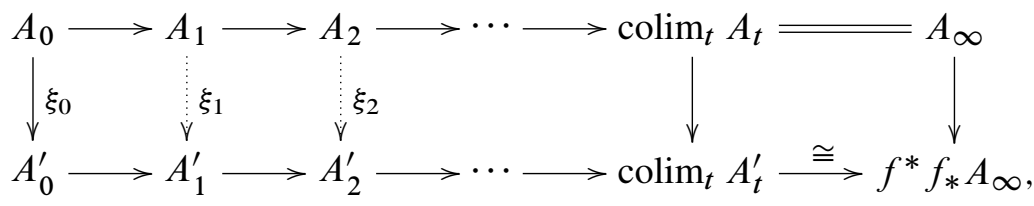

together with induced maps $\xi_{t}(t \geq 1)$ which make the diagram in SymSeq commute. By assumption we know $\xi_{0}$ is a weak equivalence, and to verify (5.6) is a weak equivalence, it is enough to check that $\xi_{t}$ is a weak equivalence for each $t \geq 1$. Since the 
horizontal maps are monomorphisms and we know $A_{t} / A_{t-1} \cong \mathcal{O}_{A}[\mathbf{t}] \check{\otimes}_{\Sigma_{t}}(Y / X)^{\check{\otimes} t}$, it is enough to verify that

$$
A \amalg(\mathcal{O} \circ(Y / X)) \longrightarrow A^{\prime} \amalg\left(\mathcal{O}^{\prime} \circ(Y / X)\right)
$$

is a weak equivalence, which is the same as verifying that

$$
Z_{0} \amalg(\mathcal{O} \circ W) \amalg(\mathcal{O} \circ(Y / X)) \longrightarrow f^{*} f_{*}\left(Z_{0} \amalg(\mathcal{O} \circ W) \amalg(\mathcal{O} \circ(Y / X))\right)
$$

is a weak equivalence. Noting that $W \amalg(Y / X)$ is cofibrant finishes the argument that (5.6) is a weak equivalence. Consider a sequence

$$
Z_{0} \longrightarrow Z_{1} \longrightarrow Z_{2} \longrightarrow \cdots
$$

of pushouts of maps as in (5.4). Assume $Z_{0}$ makes (5.5) a weak equivalence for every cofibrant $W \in$ SymSeq; we want to show that for $Z_{\infty}:=\operatorname{colim}_{k} Z_{k}$ the natural map

$$
Z_{\infty} \amalg(\mathcal{O} \circ W) \longrightarrow f^{*} f_{*}\left(Z_{\infty} \amalg(\mathcal{O} \circ W)\right)
$$

is a weak equivalence for every cofibrant $W \in$ SymSeq. Consider the diagram

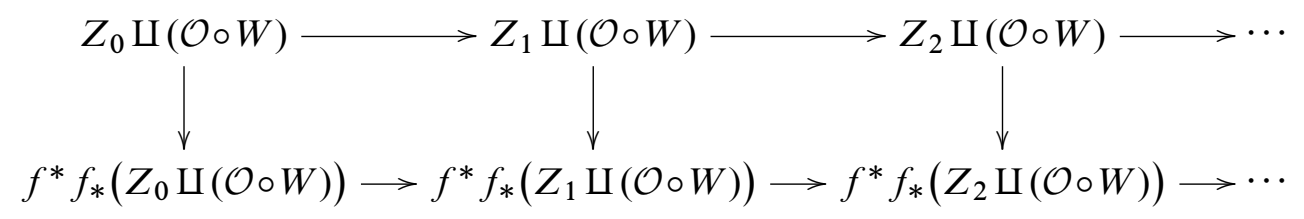

in $\mathrm{Lt}_{\mathcal{O}}$. The horizontal maps are monomorphisms and the vertical maps are weak equivalences, hence the induced map (5.7) is a weak equivalence. Noting that every cofibration $\mathcal{O} \circ * \longrightarrow Z$ in $\mathrm{Lt}_{\mathcal{O}}$ is a retract of a (possibly transfinite) composition of pushouts of maps as in (5.4), starting with $Z_{0}=\mathcal{O} \circ *$, together with Proposition 5.3, finishes the proof.

\section{Proofs}

The purpose of this section is to prove Proposition 4.28 and Proposition 4.29; we have also included a proof of Proposition 3.29 at the end of this section. First we establish a characterization of flat stable cofibrations.

\subsection{Flat stable cofibrations}

The purpose of this subsection is to prove Proposition 6.6, which identifies flat stable cofibrations in $\operatorname{SymSeq}^{G}$, for $G$ a finite group. 
It is proved in [39] that the following model category structure exists on left $\Sigma_{n}$-objects in pointed simplicial sets.

Definition 6.2 Let $n \geq 0$.

- The mixed $\Sigma_{n}$-equivariant model structure on $\mathrm{S}_{*}^{\Sigma_{n}}$ has weak equivalences the underlying weak equivalences of simplicial sets, cofibrations the retracts of (possibly transfinite) compositions of pushouts of maps

$$
\Sigma_{n} / H \cdot \partial \Delta[k]_{+} \longrightarrow \Sigma_{n} / H \cdot \Delta[k]_{+} \quad\left(k \geq 0, H \subset \Sigma_{n} \text { subgroup }\right),
$$

and fibrations the maps with the right lifting property with respect to the acyclic cofibrations.

Furthermore, it is proved in [39] that this model structure is cofibrantly generated in which the generating cofibrations and acyclic cofibrations have small domains, and that the cofibrations are the monomorphisms. It is easy to prove that the diagram category of $\left(\Sigma_{r}^{\mathrm{op}} \times G\right)$-shaped diagrams in $\mathrm{S}_{*}^{\Sigma_{n}}$ appearing in the following proposition inherits a corresponding projective model structure. This proposition, whose proof is left to the reader, will be needed for identifying flat stable cofibrations in $\mathrm{SymSeq}^{G}$.

Proposition 6.3 Let $G$ be a finite group and consider any $n, r \geq 0$. The diagram category $\left(\mathrm{S}_{*}^{\Sigma_{n}}\right)^{\Sigma_{r}^{\mathrm{op}} \times G}$ inherits a corresponding projective model structure from the mixed $\Sigma_{n}$-equivariant model structure on $\mathrm{S}_{*}^{\Sigma_{n}}$. The weak equivalences (resp. fibrations) are the underlying weak equivalences (resp. fibrations) in $S_{*}^{\Sigma_{n}}$ and the cofibrations are the monomorphisms such that $\Sigma_{r}^{\mathrm{op}} \times G$ acts freely on the simplices of the codomain not in the image.

Definition 6.4 Define $\bar{S} \in \mathrm{Sp}^{\Sigma}$ such that $\bar{S}_{n}:=S_{n}$ for $n \geq 1$ and $\bar{S}_{0}:=*$. The structure maps are the naturally occurring ones such that there exists a map of symmetric spectra $i: \bar{S} \longrightarrow S$ satisfying $i_{n}=$ id for each $n \geq 1$.

The following calculation, which follows easily from (2.9) and (2.14), will be needed for characterizing flat stable cofibrations in $\operatorname{SymSeq}^{G}$ below.

Calculation 6.5 Let $m, p \geq 0, H \subset \Sigma_{m}$ a subgroup, and $K$ a pointed simplicial set. Define $X:=G \cdot G_{p}\left(S \otimes G_{m}^{H} K\right) \in$ SymSeq $^{G}$. Here, $X$ is obtained by applying the 
indicated functors in (4.1) to $K$. Then for $r=p$ we have

$$
\begin{array}{r}
(\bar{S} \wedge X[\mathbf{r}])_{n} \cong\left\{\begin{array}{r}
G \cdot\left(\Sigma_{n} \cdot \Sigma_{n-m} \times \Sigma_{m} \bar{S}_{n-m} \wedge\left(\Sigma_{m} / H \cdot K\right)\right) \cdot \Sigma_{p} \text { for } n>m, \\
* \text { for } n \leq m,
\end{array}\right. \\
X[\mathbf{r}]_{n} \cong\left\{\begin{array}{r}
G \cdot\left(\Sigma_{n} \cdot \Sigma_{n-m} \times \Sigma_{m} S_{n-m} \wedge\left(\Sigma_{m} / H \cdot K\right)\right) \cdot \Sigma_{p} \text { for } n>m, \\
G \cdot\left(\Sigma_{m} / H \cdot K\right) \cdot \Sigma_{p} \text { for } n=m, \\
* \text { for } n<m,
\end{array}\right.
\end{array}
$$

and for $r \neq p$ we have $X[\mathbf{r}]=*=\bar{S} \wedge X[\mathbf{r}]$.

The following characterization of flat stable cofibrations in $\operatorname{SymSeq}^{G}$ is motivated by [21, Proposition 5.2.2]; we have benefitted from the discussion and corresponding characterization in [37] of cofibrations in $\mathrm{Sp}^{\Sigma}$ with the flat stable model structure.

Proposition 6.6 Let $G$ be a finite group.

(a) A map $f: X \longrightarrow Y$ in SymSeq ${ }^{G}$ with the flat stable model structure is a cofibration if and only if the induced maps

$$
\begin{array}{r}
X[\mathbf{r}]_{0} \longrightarrow Y[\mathbf{r}]_{0}, \quad r \geq 0, n=0, \\
(\bar{S} \wedge Y[\mathbf{r}])_{n} \amalg_{(\bar{S} \wedge X[\mathbf{r}])_{n}} X[\mathbf{r}]_{n} \longrightarrow Y[\mathbf{r}]_{n}, \quad r \geq 0, n \geq 1,
\end{array}
$$

are cofibrations in $\left(\mathrm{S}_{*}^{\Sigma_{n}}\right)^{\Sigma_{r}^{\mathrm{op}} \times G}$ with the model structure of Proposition 6.3.

(b) A map $f: X \longrightarrow Y$ in SymSeq ${ }^{G}$ with the positive flat stable model structure is a cofibration if and only if the maps $X[\mathbf{r}]_{0} \longrightarrow Y[\mathbf{r}]_{0}, r \geq 0$, are isomorphisms, and the induced maps

$$
(\bar{S} \wedge Y[\mathbf{r}])_{n} U_{(\bar{S} \wedge X[\mathbf{r}])_{n}} X[\mathbf{r}]_{n} \longrightarrow Y[\mathbf{r}]_{n}, \quad r \geq 0, n \geq 1,
$$

are cofibrations in $\left(\mathrm{S}_{*}^{\Sigma_{n}}\right)^{\Sigma_{r}^{\mathrm{op}} \times G}$ with the model structure of Proposition 6.3.

Proof It suffices to prove part (a). Consider any $f: X \longrightarrow Y$ in $\operatorname{SymSeq}^{G}$ with the flat stable model structure. We want a sufficient condition for $f$ to be a cofibration. The first step is to rewrite a lifting problem as a sequential lifting problem.
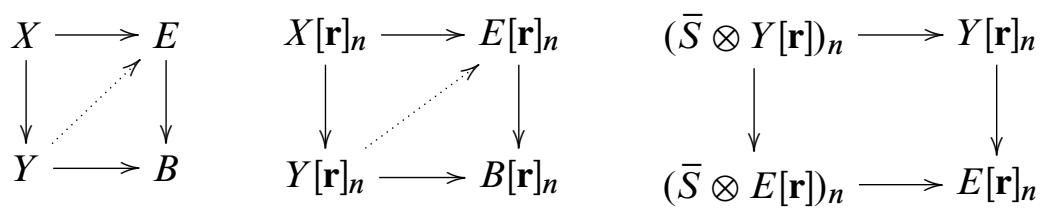
The left-hand solid commutative diagram in $\operatorname{SymSeq}^{G}$ has a lift if and only if the right-hand sequence of lifting problems in $\left(\mathrm{S}_{*}^{\Sigma_{n}}\right)^{\Sigma_{r}^{\mathrm{op}} \times G}$ has a solution, if and only if the sequence of lifting problems
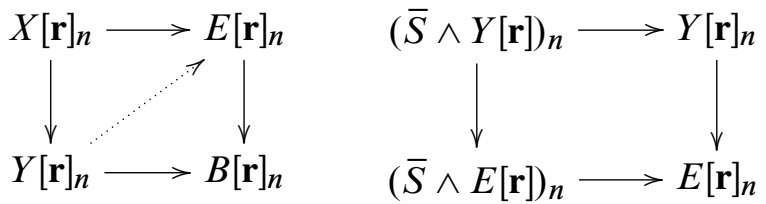

in $\left(\mathrm{S}_{*}^{\Sigma_{n}}\right)^{\Sigma_{r}^{\mathrm{op}} \times G}$ has a solution, if and only if the sequence of lifting problems
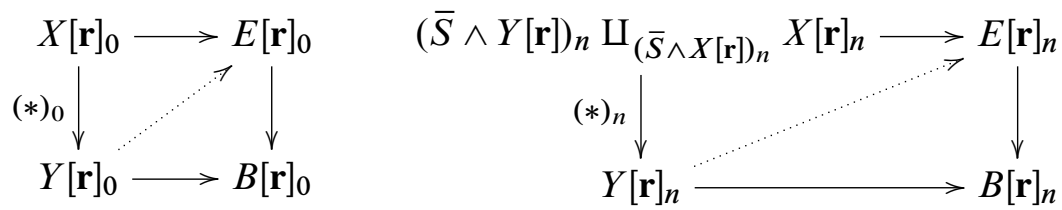

$(n \geq 1)$

has a solution. If each $(*)_{n}$ is a cofibration then $f$ has the left lifting property with respect to all acyclic fibrations, and hence $f$ is a cofibration. Conversely, suppose $f$ is a cofibration. We want to verify that each $(*)_{n}$ is a cofibration. Every cofibration is a retract of a (possibly transfinite) composition of pushouts of generating cofibrations, and hence by a reduction argument that we leave to the reader, it is sufficient to verify for $f$ a generating cofibration. Let $g: K \longrightarrow L$ be a monomorphism in $\mathrm{S}_{*}, m, p \geq 0$, $H \subset \Sigma_{m}$ a subgroup, and define $f: X \longrightarrow Y$ in $\operatorname{SymSeq}^{G}$ to be the induced map

$$
G \cdot G_{p}\left(S \otimes G_{m}^{H} K\right) \stackrel{g_{*}}{\longrightarrow} G \cdot G_{p}\left(S \otimes G_{m}^{H} L\right)
$$

Here, the map $g_{*}$ is obtained by applying the indicated functors in (4.1) to the map $g$. We know $(*)_{0}$ is a cofibration. Consider $n \geq 1$. By Calculation 6.5: $(*)_{n}$ is an isomorphism for the case $r \neq p$ and for the case $(r=p$ and $n \neq m)$. For the case $(r=p$ and $n=m),(*)_{n}$ is the map

$$
G \cdot\left(\Sigma_{m} / H \cdot K\right) \cdot \Sigma_{p} \stackrel{G \cdot\left(\Sigma_{m} / H \cdot g\right) \cdot \Sigma_{p}}{\longrightarrow} G \cdot\left(\Sigma_{m} / H \cdot L\right) \cdot \Sigma_{p}
$$

Hence in all cases $(*)_{n}$ is a cofibration. 


\subsection{Proofs}

Proof of Proposition 4.29 Consider part (b). Let $g: K \longrightarrow L$ be a monomorphism in $\mathrm{S}_{*}, m, p \geq 0, H \subset \Sigma_{m}$ a subgroup, and consider the pushout diagram

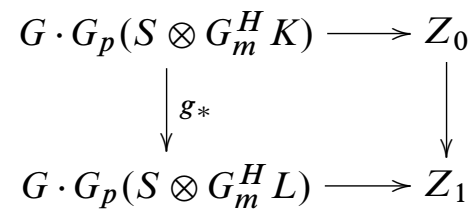

in SymSeq ${ }^{G}$. Here, the map $g_{*}$ is obtained by applying the indicated functors in (4.1) to the map $g$. Consider the functors

$$
\begin{aligned}
& -\check{\otimes}_{G} Z_{0}: \text { SymSeq }^{G^{\text {op }}} \longrightarrow \text { SymSeq, } \\
& -\check{\otimes}_{G} Z_{1}: \text { SymSeq }^{\text {op }} \longrightarrow \text { SymSeq, }
\end{aligned}
$$

and assume (6.9) preserves weak equivalences; let's verify (6.10) preserves weak equivalences. Suppose $A \longrightarrow B$ in SymSeq ${ }^{\text {op }}$ is a weak equivalence. Applying $A \check{\otimes}_{G}-$ to (6.8) gives the pushout diagram

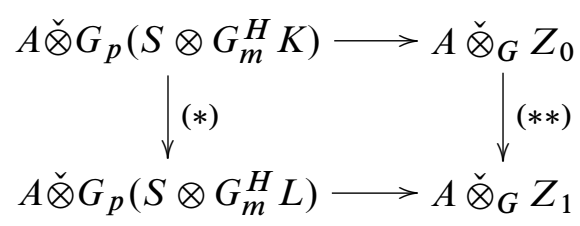

in SymSeq. Let's check $(*)$ is a monomorphism. This amounts to a calculation:

$$
\left(A \check{\otimes} G_{p}\left(S \otimes G_{m}^{H} K\right)\right)[\mathbf{r}] \cong\left\{\begin{array}{r}
A[\mathbf{r}-\mathbf{p}] \wedge\left(S \otimes G_{m}^{H} K\right) \cdot \Sigma_{r-p} \times 1 \\
\Sigma_{r} \text { for } r \geq p, \\
* \text { for } r<p .
\end{array}\right.
$$

Since the map $S \otimes G_{m}^{H} K \longrightarrow S \otimes G_{m}^{H} L$ is a cofibration in $\mathrm{Sp}^{\Sigma}$ with the flat stable model structure, smashing with any symmetric spectrum gives a monomorphism. It follows that $(*)$ is a monomorphism, and hence $(* *)$ is a monomorphism. Consider the commutative diagram

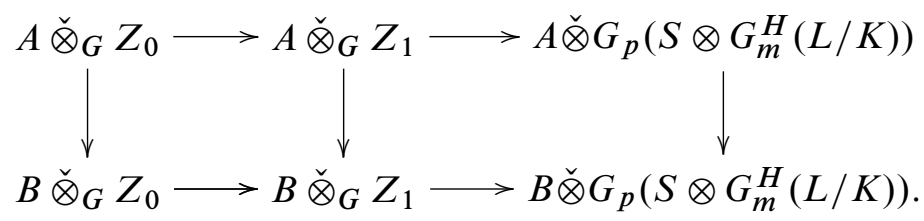

Since $S \otimes G_{m}^{H}(L / K)$ is cofibrant in $\mathrm{Sp}^{\Sigma}$ with the flat stable model structure, smashing with it preserves weak equivalences. It follows that the right-hand vertical map is a 
weak equivalence. By assumption, the left-hand vertical map is a weak equivalence, hence the middle vertical map is a weak equivalence and we get that (6.10) preserves weak equivalences. Consider a sequence

$$
Z_{0} \longrightarrow Z_{1} \longrightarrow Z_{2} \longrightarrow \cdots
$$

of pushouts of maps as in (6.8). Assume (6.9) preserves weak equivalences; we want to show that for $Z_{\infty}:=\operatorname{colim}_{k} Z_{k}$ the functor

$$
-\check{\otimes}_{G} Z_{\infty}: \text { SymSeq }^{G^{\text {op }}} \longrightarrow \text { SymSeq }
$$

preserves weak equivalences. Suppose $A \longrightarrow B$ in SymSeq ${ }^{\text {op }}$ is a weak equivalence and consider the diagram

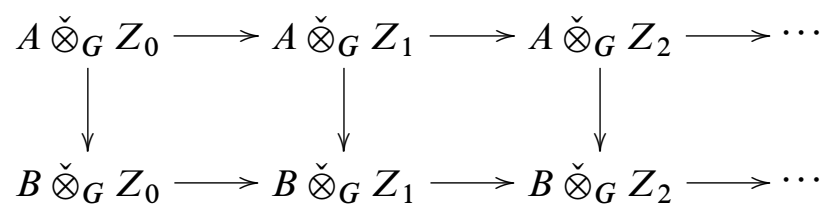

in SymSeq. The horizontal maps are monomorphisms and the vertical maps are weak equivalences, hence the induced map $A \check{\otimes}_{G} Z_{\infty} \longrightarrow B \check{\otimes}_{G} Z_{\infty}$ is a weak equivalence. Noting that every cofibration $* \longrightarrow Z$ in SymSeq ${ }^{G}$ is a retract of a (possibly transfinite) composition of pushouts of maps as in (6.8), starting with $Z_{0}=*$, finishes the proof of part (b). Consider part (a). Suppose $X \longrightarrow Y$ in SymSeq $^{G}$ is a weak equivalence between cofibrant objects; we want to show that $B \check{\otimes}_{G} X \longrightarrow B \check{\otimes}_{G} Y$ is a weak equivalence. The map $* \longrightarrow B$ factors in SymSeq $G^{\text {op }}$ as

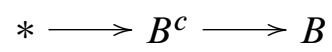

a cofibration followed by an acyclic fibration, the diagram

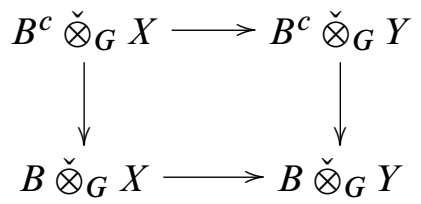

commutes, and since three of the maps are weak equivalences, so is the fourth.

Proposition 6.11 Let $G$ be a finite group. If $B \in \operatorname{SymSeq}^{G^{\mathrm{pp}}}$, then the functor

$$
B \check{\otimes}_{G}-: \text { SymSeq }^{G} \longrightarrow \text { SymSeq }
$$

sends cofibrations in $\mathrm{SymSeq}^{G}$ with the flat stable model structure to monomorphisms. 
Proof Let $g: K \longrightarrow L$ be a monomorphism in $S_{*}, m, p \geq 0, H \subset \Sigma_{m}$ a subgroup, and consider the pushout diagram

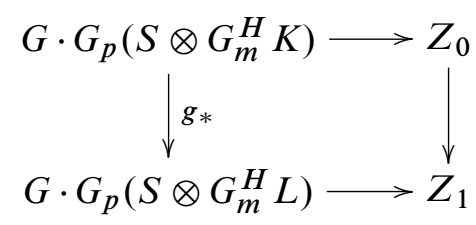

in SymSeq ${ }^{G}$. Here, the map $g_{*}$ is obtained by applying the indicated functors in (4.1) to the map $g$. Applying $B \check{\otimes}_{G}-$ gives the pushout diagram

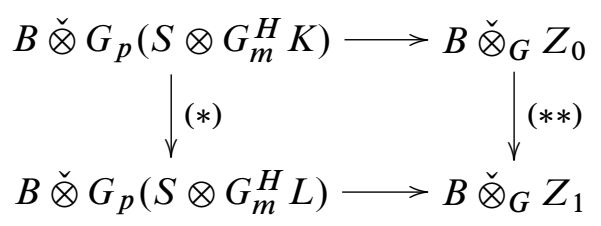

in SymSeq. The map $(*)$ is a monomorphism by the same arguments used in the proof of Proposition 4.29 , hence $(* *)$ is a monomorphism. Noting that every cofibration in SymSeq $^{G}$ is a retract of a (possibly transfinite) composition of pushouts of maps as in (6.12) completes the proof.

The following two propositions are exercises left to the reader.

Proposition 6.13 Let $t \geq 1$. Suppose the left-hand diagram is a pushout diagram in SymSeq:
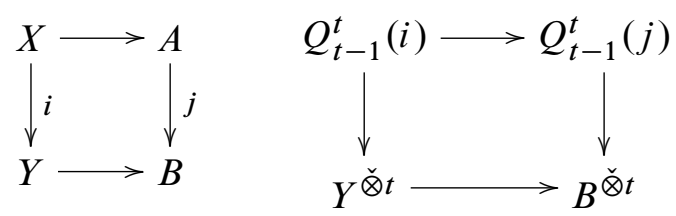

Then the corresponding right-hand diagram is a pushout diagram in SymSeq ${ }^{\Sigma_{t}}$.

Proposition 6.14 Let $t \geq 1$ and consider a commutative diagram of the form

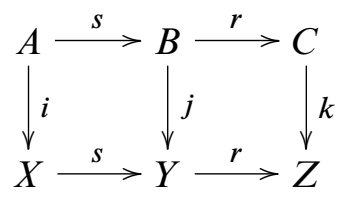


in SymSeq. Then the corresponding diagram

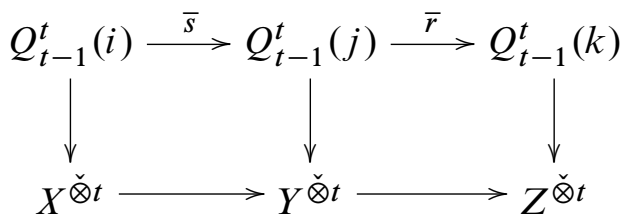

in SymSeq ${ }^{\Sigma_{t}}$ commutes. Furthermore, $\bar{r} \bar{s}=\overline{r s}$ and $\overline{\mathrm{id}}=\mathrm{id}$.

The following calculation, which follows easily from (2.9), (2.14) and (3.7), will be needed in the proof of Proposition 4.28 below.

Calculation 6.15 Let $k, m, p \geq 0, H \subset \Sigma_{m}$ a subgroup, and $t \geq 1$. Let the map $g: \partial \Delta[k]_{+} \longrightarrow \Delta[k]_{+}$be a generating cofibration for $\mathrm{S}_{*}$ and define $X \longrightarrow Y$ in SymSeq to be the induced map

$$
G_{p}\left(S \otimes G_{m}^{H} \partial \Delta[k]_{+}\right) \stackrel{g_{*}}{\longrightarrow} G_{p}\left(S \otimes G_{m}^{H} \Delta[k]_{+}\right) .
$$

Here, the map $g_{*}$ is obtained by applying the indicated functors in (4.1) to the map $g$. For $r=t p$ we have the calculation

$$
\begin{aligned}
& \left(\left(Y^{\check{\otimes} t}\right)[\mathbf{r}]\right)_{n} \cong\left\{\begin{aligned}
\Sigma_{n} \cdot \Sigma_{n-t m} \times H^{\times t} S_{n-t m} \wedge\left(\Delta[k]^{\times t}\right)_{+} \cdot \Sigma_{t p} & \text { for } n>t m, \\
\Sigma_{t m} \cdot H^{\times t}\left(\Delta[k]^{\times t}\right)_{+} \cdot \Sigma_{t p} & \text { for } n=t m, \\
& * \text { for } n<t m,
\end{aligned}\right. \\
& \left(\bar{S} \wedge\left(Y^{\check{\otimes} t}\right)[\mathbf{r}]\right)_{n} \cong\left\{\begin{aligned}
\Sigma_{n} \cdot \Sigma_{n-t m} \times H^{\times t} & \bar{S}_{n-t m} \wedge\left(\Delta[k]^{\times t}\right)_{+} \cdot \Sigma_{t p} \\
& \text { for } n>t m, \\
& \text { for } n \leq t m,
\end{aligned}\right. \\
& \left(Q_{t-1}^{t}[\mathbf{r}]\right)_{n} \cong\left\{\begin{aligned}
\Sigma_{n} \cdot \Sigma_{n-t m} \times H^{\times t} S_{n-t m} \wedge \partial\left(\Delta[k]^{\times t}\right)_{+} \cdot \Sigma_{t p} & \text { for } n>t m, \\
\Sigma_{t m} \cdot H^{\times t} \partial\left(\Delta[k]^{\times t}\right)_{+} \cdot \Sigma_{t p} & \text { for } n=t m, \\
& * \text { for } n<t m,
\end{aligned}\right. \\
& \left(\bar{S} \wedge Q_{t-1}^{t}[\mathbf{r}]\right)_{n} \cong\left\{\begin{aligned}
\Sigma_{n} \cdot \Sigma_{n-t m} \times H^{\times t} & \bar{S}_{n-t m} \wedge \partial\left(\Delta[k]^{\times t}\right)_{+} \cdot \Sigma_{t p} \text { for } n>t m, \\
* & \text { for } n \leq t m,
\end{aligned}\right.
\end{aligned}
$$

and for $r \neq t p$ we have $\left(Y^{\check{\otimes} t}\right)[\mathbf{r}]=*=\bar{S} \wedge\left(Y^{\check{\otimes} t}\right)[\mathbf{r}]$ and $Q_{t-1}^{t}[\mathbf{r}]=*=\bar{S} \wedge Q_{t-1}^{t}[\mathbf{r}]$.

The following proposition is proved in [3, I.2] and will be useful below for verifying that certain induced maps are cofibrations. 
Proposition 6.16 Let $\mathrm{M}$ be a model category and consider a commutative diagram of the form

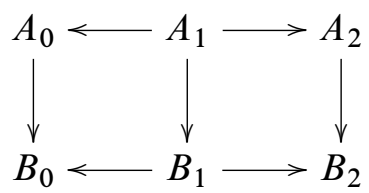

in M. If the maps $A_{0} \longrightarrow B_{0}$ and $B_{1} \amalg_{A_{1}} A_{2} \longrightarrow B_{2}$ are cofibrations, then the induced map

$$
A_{0} \amalg_{A_{1}} A_{2} \longrightarrow B_{0} \amalg_{B_{1}} B_{2}
$$

is a cofibration.

Proof of Proposition 4.28 Consider part (a). The argument is by induction on $t$. Let $m \geq 1, H \subset \Sigma_{m}$ a subgroup, and $k, p \geq 0$. Let $g: \partial \Delta[k]_{+} \longrightarrow \Delta[k]_{+}$be a generating cofibration for $S_{*}$ and consider the pushout diagram

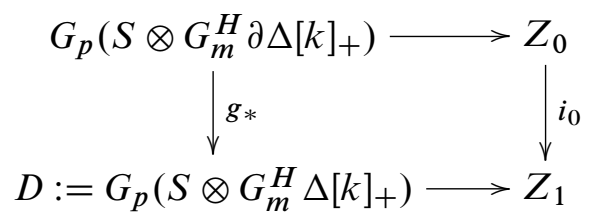

in SymSeq with $Z_{0}$ cofibrant. Here, the map $g_{*}$ is obtained by applying the indicated functors in (4.1) to the map $g$. By Proposition 6.13, the corresponding diagram

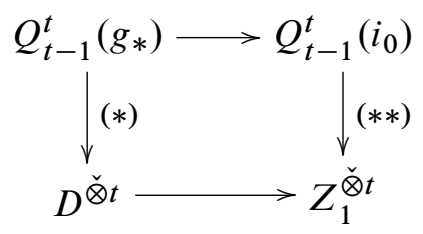

is a pushout diagram in SymSeq ${ }^{\Sigma_{t}}$. Since $m \geq 1$, it follows from Proposition 6.6 and Calculation 6.15 that $(*)$ is a cofibration in SymSeq ${ }^{\Sigma_{t}}$, and hence $(* *)$ is a cofibration. Consider a sequence

$$
Z_{0} \stackrel{i_{0}}{\longrightarrow} Z_{1} \stackrel{i_{1}}{\longrightarrow} Z_{2} \stackrel{i_{2}}{\longrightarrow} \cdots
$$

of pushouts of maps as in (6.17), define $Z_{\infty}:=\operatorname{colim}_{q} Z_{q}$, and consider the naturally occurring map $i_{\infty}: Z_{0} \longrightarrow Z_{\infty}$. Using Proposition 6.16 and (4.14), it is easy to verify that each $Z_{q}^{\ddot{\otimes} t} \longrightarrow Q_{t-1}^{t}\left(i_{q}\right)$ is a cofibration in SymSeq ${ }^{\Sigma_{t}}$. By above we know that each $Q_{t-1}^{t}\left(i_{q}\right) \stackrel{\longrightarrow}{\longrightarrow} Z_{q+1}^{\check{\otimes} t}$ is a cofibration. It follows immediately that each $Z_{q}^{\check{\otimes} t} \longrightarrow Z_{q+1}^{\check{\otimes} t}$ is a cofibration in SymSeq ${ }^{\Sigma_{t}}$, and hence the map $Z_{0}^{\check{\otimes} t} \longrightarrow Z_{\infty}^{\check{\otimes} t}$ is a cofibration. Noting 
that every cofibration between cofibrant objects in SymSeq with the positive flat stable model structure is a retract of a (possibly transfinite) composition of pushouts of maps as in (6.17) finishes the proof for part (a). Consider part (b). Proceed as above for part (a) and consider the commutative diagram

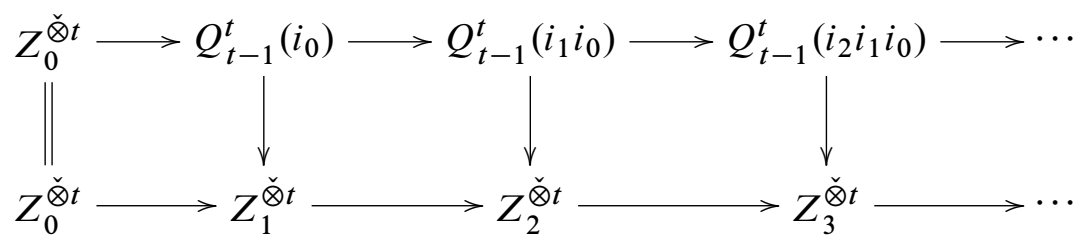

in SymSeq ${ }^{\Sigma_{t}}$. We claim that (6.19) is a diagram of cofibrations. By part (a), the bottom row is a diagram of cofibrations. Using Proposition 6.16 and (4.14), it is easy to verify that if $i$ and $j$ are composable cofibrations between cofibrant objects in SymSeq, then the induced maps

$$
Q_{t-1}^{t}(i) \longrightarrow Q_{t-1}^{t}(j i) \longrightarrow Q_{t-1}^{t}(j)
$$

are cofibrations in SymSeq ${ }^{\Sigma_{t}}$; it follows easily that the vertical maps and the top row maps are cofibrations. Applying $B \check{\otimes}_{\Sigma_{t}}-$ to (6.19) gives the commutative diagram

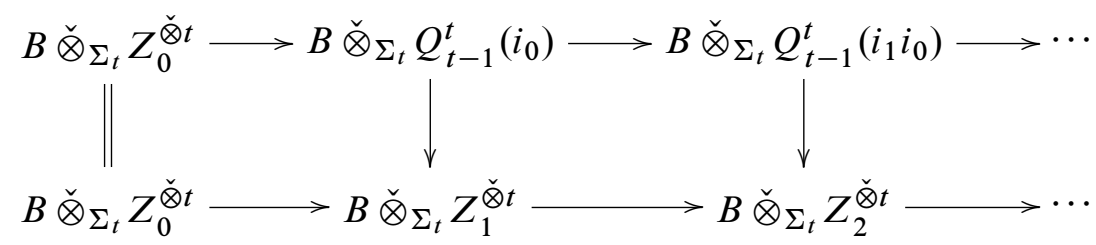

in SymSeq. By Proposition 6.11, (6.20) is a diagram of monomorphisms, hence the induced map $B \check{\otimes}_{\Sigma_{t}} Q_{t-1}^{t}\left(i_{\infty}\right) \longrightarrow B \check{\otimes}_{\Sigma_{t}} Z_{\infty}^{\check{\otimes} t}$ is a monomorphism. Noting that every cofibration between cofibrant objects in SymSeq is a retract of a (possibly transfinite) composition of pushouts of maps as in (6.17), together with Proposition 6.14, finishes the proof for part (b).

Proof of Proposition 3.29 Suppose $A: \mathrm{D} \longrightarrow \mathrm{Lt}_{\mathcal{O}}$ is a small diagram. We want to show that colim $A$ exists. It is easy to verify, using Proposition 4.10, that this colimit may be calculated by a reflexive coequalizer in $L_{\mathcal{O}}$ of the form

$$
\operatorname{colim} A \cong \operatorname{colim}\left(\operatorname{colim}_{d \in \mathrm{D}}\left(\mathcal{O} \circ A_{d}\right) \underset{(\mathrm{id} \circ m)_{*}}{(m \circ \mathrm{id})_{*}} \operatorname{colim}\left(\mathcal{O} \circ \mathcal{O} \circ A_{d}\right)\right),
$$

provided that the indicated colimits appearing in this reflexive pair exist in $\mathrm{Lt}_{\mathcal{O}}$. The underlying category SymSeq has all small colimits, and left adjoints preserve colimiting 
cones, hence there is a commutative diagram

$$
\begin{aligned}
& \underset{d \in \mathrm{D}}{\operatorname{colim}}\left(\mathcal{O} \circ A_{d}\right) \underset{(\mathrm{id} \circ m)_{*}}{(m \circ \mathrm{id})_{*}} \operatorname{colim}\left(\mathcal{O} \circ \mathcal{O} \circ A_{d}\right) \\
& \downarrow \cong \downarrow \\
& \mathcal{O} \circ\left(\operatorname{colim}_{d \in D} A_{d}\right) \leftleftarrows \mathcal{O} \circ\left(\underset{d \in D}{\operatorname{colim}}\left(\mathcal{O} \circ A_{d}\right)\right)
\end{aligned}
$$

in $\mathrm{Lt}_{\mathcal{O}}$; the colimits in the bottom row exist since they are in the underlying category SymSeq (we have dropped the notation for the forgetful functor $U$ ), hence the colimits in the top row exist in $\mathrm{Lt}_{\mathcal{O}}$. Therefore colim $A$ exists and Proposition 3.27 completes the proof.

\section{Constructions in the special case of algebras over an op- erad}

Some readers may only be interested in the special case of algebras over an operad and may wish to completely avoid working with the circle product and the left $\mathcal{O}_{-}$ module constructions. It is easy to translate the constructions and proofs in this paper into the special case of algebras while avoiding the circle product notation. Usually, this amounts to replacing (SymSeq, $\check{\otimes})$ with $\left(\mathrm{Sp}^{\Sigma}, \wedge\right)$, replacing the left adjoint $\mathcal{O} \circ-:$ SymSeq $\longrightarrow \mathrm{Lt}_{\mathcal{O}}$ with the left adjoint $\mathcal{O}(-): \mathrm{Sp}^{\Sigma} \longrightarrow \mathrm{Alg}_{\mathcal{O}}$ (Definition 3.17), and then replacing the symmetric array $\mathcal{O}_{A}$ in Proposition 4.7 with the symmetric sequence $\mathcal{O}_{A}$ in Proposition 7.2. We illustrate below with several special cases of particular interest.

\subsection{Special cases}

Proposition 4.7 has the following special case.

Proposition 7.2 Let $\mathcal{O}$ be an operad in symmetric spectra, $A \in \mathrm{Alg}_{\mathcal{O}}$, and $Y \in \mathrm{Sp}^{\Sigma}$. Consider any coproduct in $\mathrm{Alg}_{\mathcal{O}}$ of the form

$$
A \amalg \mathcal{O}(Y) .
$$

There exists a symmetric sequence $\mathcal{O}_{A}$ and natural isomorphisms

$$
A \amalg \mathcal{O}(Y) \cong \coprod_{q \geq 0} \mathcal{O}_{A}[\mathbf{q}] \wedge \Sigma_{q} Y^{\wedge q}
$$


in the underlying category $\mathrm{Sp}^{\Sigma}$. If $q \geq 0$, then $\mathcal{O}_{A}[\mathbf{q}]$ is naturally isomorphic to a colimit of the form

$$
\mathcal{O}_{A}[\mathbf{q}] \cong \operatorname{colim}\left(\coprod_{p \geq 0} \mathcal{O}[\mathbf{p}+\mathbf{q}] \wedge \Sigma_{p} A^{\wedge p} \underset{d_{0}}{\longleftarrow} \coprod_{p \geq 0} \mathcal{O}[\mathbf{p}+\mathbf{q}] \wedge \Sigma_{\Sigma_{p}}(\mathcal{O}(A))^{\wedge p}\right),
$$

in $\mathrm{Sp}^{\Sigma}$, with $d_{0}$ induced by operad multiplication and $d_{1}$ induced by $m: \mathcal{O}(A) \longrightarrow A$.

Definition 4.13 has the following special case.

Definition 7.4 Let $i: X \longrightarrow Y$ be a morphism in $\mathrm{Sp}^{\Sigma}$ and $t \geq 1$. Define $Q_{0}^{t}:=X^{\wedge t}$ and $Q_{t}^{t}:=Y^{\wedge t}$. For $0<q<t$ define $Q_{q}^{t}$ inductively by the pushout diagrams

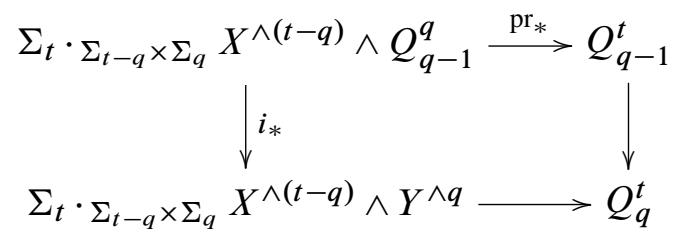

in $\left(\mathrm{Sp}^{\Sigma}\right)^{\Sigma_{t}}$. We sometimes denote $Q_{q}^{t}$ by $Q_{q}^{t}(i)$ to emphasize in the notation the map $i: X \longrightarrow Y$. The maps $\mathrm{pr}_{*}$ and $i_{*}$ are the obvious maps induced by $i$ and the appropriate projection maps.

Proposition 4.20 has the following special case.

Proposition 7.5 Let $\mathcal{O}$ be an operad in symmetric spectra, $A \in \operatorname{Alg}_{\mathcal{O}}$, and $i: X \longrightarrow Y$ in $\mathrm{Sp}^{\Sigma}$. Consider any pushout diagram in $\mathrm{Alg}_{\mathcal{O}}$ of the form

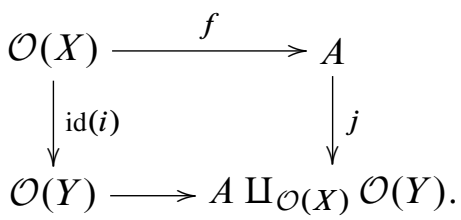

The pushout in (7.6) is naturally isomorphic to a filtered colimit of the form

$$
A \amalg_{\mathcal{O}(X)} \mathcal{O}(Y) \cong \operatorname{colim}\left(A_{0} \stackrel{j_{1}}{\longrightarrow} A_{1} \stackrel{j_{2}}{\longrightarrow} A_{2} \stackrel{j_{3}}{\longrightarrow} \cdots\right)
$$


in the underlying category $\mathrm{Sp}^{\Sigma}$, with $A_{0}:=\mathcal{O}_{A}[\mathbf{0}] \cong A$ and $A_{t}$ defined inductively by pushout diagrams in $\mathrm{Sp}^{\Sigma}$ of the form:

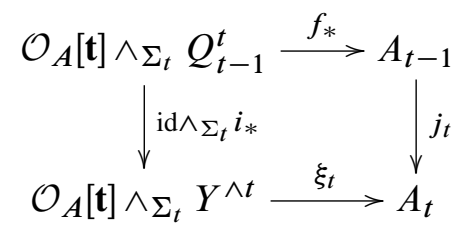

Propositions 4.28, 4.29 and 4.30 have the following special cases, respectively.

Proposition 7.7 Let $B \in\left(\mathrm{Sp}^{\Sigma}\right)^{\Sigma_{t}^{\mathrm{op}}}$ and $t \geq 1$. If $i: X \longrightarrow Y$ is a cofibration between cofibrant objects in $\mathrm{Sp}^{\Sigma}$ with the positive flat stable model structure, then

(a) $X^{\wedge t} \longrightarrow Y^{\wedge t}$ is a cofibration in $\left(\mathrm{Sp}^{\Sigma}\right)^{\Sigma_{t}}$ with the positive flat stable model structure, and hence with the flat stable model structure,

(b) the map $B \wedge \Sigma_{t} Q_{t-1}^{t} \longrightarrow B \wedge_{\Sigma_{t}} Y^{\wedge t}$ is a monomorphism.

Proposition 7.8 Let $G$ be a finite group and consider $\mathrm{Sp}^{\Sigma},\left(\mathrm{Sp}^{\Sigma}\right)^{G}$, and $\left(\mathrm{Sp}^{\Sigma}\right)^{G^{\mathrm{op}}}$ each with the flat stable model structure.

(a) If $B \in\left(\mathrm{Sp}^{\Sigma}\right)^{G^{\mathrm{op}}}$, then the functor

$$
B \wedge_{G}-:\left(\mathrm{Sp}^{\Sigma}\right)^{G} \longrightarrow \mathrm{Sp}^{\Sigma}
$$

preserves weak equivalences between cofibrant objects, and hence its total left derived functor exists.

(b) If $Z \in\left(\mathrm{Sp}^{\Sigma}\right)^{G}$ is cofibrant, then the functor

$$
-\wedge_{G} Z:\left(\mathrm{Sp}^{\Sigma}\right)^{G^{\mathrm{op}}} \longrightarrow \mathrm{Sp}^{\Sigma}
$$

preserves weak equivalences.

Proposition 7.9 If the map $i: X \longrightarrow Y$ in Proposition 7.5 is a generating acyclic cofibration in $\mathrm{Sp}^{\Sigma}$ with the positive flat stable model structure, then each map $j_{t}$ is a monomorphism and a weak equivalence. In particular, the map $j$ is a monomorphism and a weak equivalence. 


\section{References}

[1] M Basterra, M A Mandell, Homology and cohomology of $E_{\infty}$ ring spectra, Math. Z. 249 (2005) 903-944 MR2126222

[2] C Berger, I Moerdijk, Axiomatic homotopy theory for operads, Comment. Math. Helv. 78 (2003) 805-831 MR2016697

[3] W Chachólski, J Scherer, Homotopy theory of diagrams, Mem. Amer. Math. Soc. 155 (2002) x+90 MR1879153

[4] W G Dwyer, J Spaliński, Homotopy theories and model categories, from: "Handbook of algebraic topology”, (I M James, editor), North-Holland, Amsterdam (1995) 73-126 MR1361887

[5] A D Elmendorf, I Kriz, M A Mandell, J P May, Rings, modules, and algebras in stable homotopy theory, Math. Surveys and Monogr. 47, Amer. Math. Soc. (1997) MR1417719 With an appendix by M Cole

[6] A D Elmendorf, MA Mandell, Rings, modules, and algebras in infinite loop space theory, Adv. Math. 205 (2006) 163-228 MR2254311

[7] B Fresse, Lie theory of formal groups over an operad, J. Algebra 202 (1998) 455-511 MR1617616

[8] B Fresse, Koszul duality of operads and homology of partition posets, from: "Homotopy theory: relations with algebraic geometry, group cohomology, and algebraic $K$-theory", (P G Goerss, S Priddy, editors), Contemp. Math. 346, Amer. Math. Soc. (2004) 115-215 MR2066499

[9] B Fresse, Modules over operads and functors, Lecture Notes in Math. 1967, Springer, Berlin (2009) MR2494775

[10] E Getzler, J D S Jones, Operads, homotopy algebra and iterated integrals for double loop spaces arXiv:hep-th/9403055v1

[11] V Ginzburg, M Kapranov, Koszul duality for operads, Duke Math. J. 76 (1994) 203-272 MR1301191

[12] P G Goerss, M J Hopkins, André-Quillen (co)-homology for simplicial algebras over simplicial operads, from: "Une dégustation topologique [Topological morsels]: homotopy theory in the Swiss Alps (Arolla, 1999)", (D Arlettaz, K Hess, editors), Contemp. Math. 265, Amer. Math. Soc. (2000) 41-85 MR1803952

[13] P G Goerss, M J Hopkins, Moduli spaces of commutative ring spectra, from: "Structured ring spectra”, (A Baker, B Richter, editors), London Math. Soc. Lecture Note Ser. 315, Cambridge Univ. Press (2004) 151-200 MR2125040

[14] P G Goerss, M J Hopkins, Moduli problems for structured ring spectra (2005) Available at http://hopf.math.purdue.edu 
[15] PG Goerss, J F Jardine, Simplicial homotopy theory, Progress in Math. 174, Birkhäuser Verlag, Basel (1999) MR1711612

[16] J E Harper, Homotopy theory of modules over operads and non- $\Sigma$ operads in monoidal model categories arXiv:0801.0191

[17] V Hinich, Homological algebra of homotopy algebras, Comm. Algebra 25 (1997) 3291-3323 MR1465117

[18] V Hinich, V Schechtman, Homotopy Lie algebras, from: "I M Gel' fand Seminar", (S $\mathrm{Gel}^{\prime}$ fand, S Gindikin, editors), Adv. Soviet Math. 16, Amer. Math. Soc. (1993) 1-28 MR1237833

[19] PS Hirschhorn, Model categories and their localizations, Math. Surveys and Monogr. 99, Amer. Math. Soc. (2003) MR1944041

[20] M Hovey, Model categories, Math. Surveys and Monogr. 63, Amer. Math. Soc. (1999) MR1650134

[21] M Hovey, B Shipley, J Smith, Symmetric spectra, J. Amer. Math. Soc. 13 (2000) 149-208 MR1695653

[22] M Kapranov, Y Manin, Modules and Morita theorem for operads, Amer. J. Math. 123 (2001) 811-838 MR1854112

[23] G M Kelly, On the operads of JP May, Repr. Theory Appl. Categ. (2005) 1-13 MR2177746

[24] I Kříž, J P May, Operads, algebras, modules and motives, Astérisque (1995) iv+145pp MR1361938

[25] L G Lewis, Jr, MA Mandell, Modules in monoidal model categories, J. Pure Appl. Algebra 210 (2007) 395-421 MR2320005

[26] S Mac Lane, Categories for the working mathematician, second edition, Graduate Texts in Math. 5, Springer, New York (1998) MR1712872

[27] M A Mandell, $E_{\infty}$ algebras and p-adic homotopy theory, Topology 40 (2001) 43-94 MR1791268

[28] M A Mandell, J P May, S Schwede, B Shipley, Model categories of diagram spectra, Proc. London Math. Soc. (3) 82 (2001) 441-512 MR1806878

[29] M Markl, S Shnider, J Stasheff, Operads in algebra, topology and physics, Math. Surveys and Monogr. 96, Amer. Math. Soc. (2002) MR1898414

[30] J P May, The geometry of iterated loop spaces, Lectures Notes in Math. 271, Springer, Berlin (1972) MR0420610

[31] J E McClure, J H Smith, A solution of Deligne's Hochschild cohomology conjecture, from: "Recent progress in homotopy theory (Baltimore, MD, 2000)", (D M Davis, J Morava, G Nishida, W S Wilson, N Yagita, editors), Contemp. Math. 293, Amer. Math. Soc. (2002) 153-193 MR1890736 
[32] J E McClure, J H Smith, Operads and cosimplicial objects: an introduction, from: "Axiomatic, enriched and motivic homotopy theory", (J P C Greenless, editor), NATO Sci. Ser. II Math. Phys. Chem. 131, Kluwer Acad. Publ., Dordrecht (2004) 133-171 MR2061854

[33] D G Quillen, Homotopical algebra, Lecture Notes in Math. 43, Springer, Berlin (1967) MR0223432

[34] D G Quillen, Rational homotopy theory, Ann. of Math. (2) 90 (1969) 205-295 MR0258031

[35] C Rezk, Spaces of algebra structures and cohomology of operads, PhD thesis, Massachusetts Institute of Technology (1996) Available at http://www.math.uiuc.edu/ r rezk

[36] S Schwede, S-modules and symmetric spectra, Math. Ann. 319 (2001) 517-532 MR1819881

[37] S Schwede, An untitled book project about symmetric spectra (2007) Available at http://www . math.uni-bonn.de/people/schwede/

[38] S Schwede, B Shipley, Algebras and modules in monoidal model categories, Proc. London Math. Soc. (3) 80 (2000) 491-511 MR1734325

[39] B Shipley, A convenient model category for commutative ring spectra, from: "Homotopy theory: relations with algebraic geometry, group cohomology, and algebraic $K$-theory”, (P G Goerss, S Priddy, editors), Contemp. Math. 346, Amer. Math. Soc. (2004) 473-483 MR2066511

[40] V A Smirnov, Homotopy theory of coalgebras, Izv. Akad. Nauk SSSR Ser. Mat. 49 (1985) 1302-1321, 1343 MR816858

[41] M Spitzweck, Operads, algebras and modules in general model categories arXiv: math/0101102v1

Institut de Géométrie, Algèbre et Topologie, EPFL

CH-1015 Lausanne, Switzerland

Department of Mathematics, University of Notre Dame

Notre Dame, IN 46556, USA

john.edward.harper@gmail.com

Received: 2 January 2008 Revised: 18 October 2008 